\title{
COLLAPSE OF THE KELDYSH CHAINS AND STABILITY OF CONTINUOUS NON-CONSERVATIVE SYSTEMS
}

\author{
O.N. Kirillov 1 and A.P. Seyranian \\ Institute of Mechanics, Moscow State Lomonosov University \\ Michurinsky pr. 1, 119192 Moscow, Russia \\ E-mail: kirillov@imec.msu.ru, seyran@imec.msu.ru
}

\begin{abstract}
In the present paper eigenvalue problems for non-selfadjoint linear differential operators smoothly dependent on a vector of real parameters are considered. Bifurcation of eigenvalues along smooth curves in the parameter space is studied. The case of a multiple eigenvalue with the Keldysh chain of arbitrary length is investigated. Explicit expressions describing bifurcation of eigenvalues are found. The obtained formulae use eigenfunctions and associated functions of the adjoint eigenvalue problems as well as the derivatives of the differential operator taken at the initial point of the parameter space. These results are important for the stability theory, sensitivity analysis and structural optimization. As a mechanical application the extended Beck problem of stability of an elastic column subjected to the partially tangential follower force, the Smith-Herrmann problem as well as the problem of flutter instability of a rectangular panel at high Mach numbers are considered and discussed in detail.
\end{abstract}

\section{Introduction}

Non-selfadjoint operators appear in non-conservative problems of mechanics and physics. In the discrete problems such operator is just a nonsymmetrical matrix. It is known that in the generic case the spectrum of a multiparameter family of nonsymmetrical matrices contains multiple eigenvalues with the Jordan chains [22]. It turns out that such eigenvalues define geometrical properties of the stability boundary of a corresponding non-conservative system [22]. An effective tool of analysis of this boundary is the study of a bifurcation of eigenvalues due to change of parameters [2, 3, 12]. From the point of view of applications this is a basis for the sensitivity analysis of structures, which in the case of simple eigenvalues was developed in [19]. To do the sensitivity analysis in continuous case we need to study bifurcations of eigenvalues in multiparameter families of non-selfadjoint differential operators.

\footnotetext{
${ }^{1}$ Visiting the Department of Solid Mechanics, The Technical University of Denmark, Lyngby, Denmark.
} 
The general theory of non-selfadjoint operators ascending to works by G. Birkhoff was then developed by many mathematicians. M.V. Keldysh was the first who generalized the notion of the Jordan chain of vectors to a wide class of non-selfadjoint operators [1]. For that reason it was called the Keldysh chain [7]. In the work [6] the perturbation theory for nonsymmetrical matrices and non-selfadjoint operators smoothly dependent on one parameter was developed. This theory allows to find the perturbation coefficients of eigenvalues and eigenvectors. The study of generic properties of the spectrum of the multiparameter family of non-selfadjoint differential operators remains difficult problem. It seems that in the infinite dimensional case there is still no analogue to the Arnold theory of versal deformations of matrices, allowing to classify the generic singularities of the bifurcation and stability diagrams: even in the selfadjoint case the progress is quite slow [13].

In our paper we combine the ideas of [1], [6] and [2] together. This allows us to find the explicit formulae describing bifurcation of multiple eigenvalues with the Keldysh chain of any length. These formulae suit for a wide class of nonselfadjoint eigenvalue problems arising in applications and take into account parameters both in the differential expression and in the boundary conditions. Besides, our approach allows to study multiple eigenvalues both in regular and degenerate cases. Analogous approach was applied recently to the investigation of stability problems for continuous conservative systems with gyroscopic forces [4].

\section{Basic relations}

Consider an eigenvalue problem for a linear differential operator $L$ (using the notation of M.A. Naimark [5]) defined by

$$
l(u)=\lambda u, \quad U^{s}(u)=0, \quad s=1, \ldots, m,
$$

where

$$
\begin{gathered}
l(u) \equiv \sum_{i=0}^{m} a_{i} \frac{d^{m-i} u}{d x^{m-i}}, \\
U^{s}(u) \equiv \sum_{i=0}^{m-1}\left(\left.\alpha_{i}^{s} \frac{d^{i} u}{d x^{i}}\right|_{x=0}+\left.\beta_{i}^{s} \frac{d^{i} u}{d x^{i}}\right|_{x=1}\right) .
\end{gathered}
$$

The operators $U^{s}(u)$ are linear forms with respect to the variables $u(0), u^{\prime}(0), \ldots, u^{(m-1)}(0) ; u(1)$, $u^{\prime}(1), \ldots, u^{(m-1)}(1)$. These variables are values of the function $u \in C^{(m)}[0,1]$ and its derivatives up to $(m-1)$ - th order evaluated at the points $x=0$ and $x=1$. It is assumed that the forms $U^{s}$, $s=1,2, \ldots, m$ are linearly independent. 
The differential expression

$$
l^{*}(v) \equiv \sum_{i=0}^{m}(-1)^{m-i} \overline{a_{i}} \frac{d^{m-i} v}{d x^{m-i}},
$$

where the overbar denotes complex conjugation, is called adjoint to the differential expression $l(u)[5]$. With the use of integration by parts it can be shown that

$$
\int_{0}^{1} l(u) \bar{v} d x=P(\alpha, \beta)+\int_{0}^{1} u \overline{l^{*}(v)} d x,
$$

where $P(\alpha, \beta)$ - is a bilinear form of variables

$$
\begin{aligned}
& \alpha=\left(u(0), u^{\prime}(0), \ldots, u^{(m-1)}(0), u(1), u^{\prime}(1), \ldots, u^{(m-1)}(1)\right) \\
& \beta=\left(v(0), v^{\prime}(0), \ldots, v^{(m-1)}(0), v(1), v^{\prime}(1), \ldots, v^{(m-1)}(1)\right) .
\end{aligned}
$$

Let us choose the forms $U^{m+1}, U^{m+2}, \ldots, U^{2 m}$ so that $U^{1}, U^{2}, \ldots, U^{2 m}$ be linearly independent. Then variables (3) can be expressed as linear combinations of the forms $U^{1}, U^{2}, \ldots, U^{2 m}$. Substituting these linear combinations into (2), we get the Lagrange identity [5]

$$
(l(u), v)-\left(u, l^{*}(v)\right)=U^{1} V^{2 m}+\cdots+U^{2 m} V^{1},
$$

where $(u, v)=\int_{0}^{1} u(x) \bar{v}(x) d x$ is the scalar product of functions $u, v \in C^{m}[0,1]$.

The coefficients at $U^{1}, U^{2}, \ldots, U^{2 m}$ are linear forms with respect to variables (4), and are denoted by $V^{2 m}, \ldots, V^{2}, V^{1}$, respectively. The forms $V^{1}, V^{2}, \ldots, V^{2 m}$ are linearly independent [5]. The boundary conditions

$$
V^{s}(v)=0, \quad s=1, \ldots, m
$$

are called adjoint to boundary conditions (11). The differential operator $L^{*}$, corresponding to the differential expression $l^{*}(v)$ and to the adjoint boundary conditions, is called adjoint to the operator $L$, and we say that the eigenvalue problem

$$
l^{*}(v)=\bar{\lambda} v, \quad V^{s}(v)=0, \quad s=1, \ldots, m,
$$

is adjoint to eigenvalue problem (1).

Due to boundary conditions (11), (6) identity (5) for the adjoint operators $L$ and $L^{*}$ takes a simple form: $(l(u), v)=\left(u, l^{*}(v)\right)$. If we consider differential expression $l(u)$ and assume that the function $u$ satisfies the non-homogeneous boundary conditions

$$
U^{s}(u)=G^{s}, \quad s=1,2, \ldots, m,
$$


then the Lagrange identity (5) is

$$
(l(u), v)-\left(u, l^{*}(v)\right)=G^{1} V^{2 m}+\cdots+G^{m} V^{m+1} .
$$

This is valid since $v$ satisfies boundary conditions (6).

\section{Collapse of the Keldysh chain}

Suppose that in eigenvalue problem (1) the coefficients of the differential expression $l(u)$ and the coefficients of the forms $U^{s}(u)$ are real functions, smoothly dependent on a vector of real parameters $\mathbf{p}=\left(p_{1}, p_{2}, \ldots, p_{n}\right)$, i.e. are $C^{\infty}$ - functions on an open set $\Omega \subset R^{n}$. Let $\lambda_{0}$ be an eigenvalue of the operator $L$ at the point $\mathbf{p}=\mathbf{p}_{0}$. We are interested in bifurcation of eigenvalues along the curves $\mathbf{p}(\epsilon)=\mathbf{p}_{0}+\epsilon \mathbf{e}+\epsilon^{2} \mathbf{d}+o\left(\epsilon^{2}\right)$, emitted from the initial point $\mathbf{p}_{0}$ in the parameter space. The vector $\mathbf{e}=\left(e_{1}, e_{2}, \ldots, e_{n}\right)$ defines the direction of a curve, and $\epsilon \geq 0$ is a small parameter.

Due to variation of parameters the differential expression $l(u)$ and the forms $U^{s}(u)$ take increments

$$
l(u)=l_{0}(u)+\epsilon l_{1}(u)+\epsilon^{2} l_{2}(u)+\ldots, \quad U^{s}(u)=U_{0}^{s}(u)+\epsilon U_{1}^{s}(u)+\epsilon^{2} U_{2}^{s}(u)+\ldots,
$$

where $l_{0}=\left.l(u)\right|_{\mathbf{p}=\mathbf{p}_{0}}, U_{0}^{s}=\left.U^{s}(u)\right|_{\mathbf{p}=\mathbf{p}_{0}}$, the differential expressions $l_{1}(u), l_{2}(u)$ look like

$$
l_{1}(u)=\sum_{i=1}^{n} e_{i} \frac{\partial l}{\partial p_{i}}(u), \quad l_{2}(u)=\sum_{i=1}^{n} d_{i} \frac{\partial l}{\partial p_{i}}(u)+\frac{1}{2} \sum_{i, j=1}^{n} e_{i} e_{j} \frac{\partial^{2} l}{\partial p_{i} \partial p_{j}}(u)
$$

and for the forms $U_{1}^{s}(u), U_{2}^{s}(u)$ we have

$$
U_{1}^{s}(u)=\sum_{i=1}^{n} e_{i} \frac{\partial U^{s}}{\partial p_{i}}(u), \quad U_{2}^{s}(u)=\sum_{i=1}^{n} d_{i} \frac{\partial U^{s}}{\partial p_{i}}(u)+\frac{1}{2} \sum_{i, j=1}^{n} e_{i} e_{j} \frac{\partial^{2} U^{s}}{\partial p_{i} \partial p_{j}}(u) .
$$

All the derivatives in formulae (10), (11) are evaluated at the point $\mathbf{p}=\mathbf{p}_{0}$. Thus, we deal with the regular perturbations which do not increase the order of the non-perturbed operator $L_{0}=L\left(\mathbf{p}_{0}\right)$ [6].

Consider an eigenvalue $\lambda_{0}$ with the Keldysh chain of length $k>0$. This means that at $\mathbf{p}=\mathbf{p}_{0}$ there exist an eigenfunction $u_{0}(x)$ and associated functions $u_{1}(x), u_{2}(x), \ldots, u_{k-1}(x)$, corresponding to the $\lambda_{0}$ and satisfying the equations and the boundary conditions

$$
\begin{gathered}
l_{0}\left(u_{0}\right)=\lambda_{0} u_{0}, \quad U_{0}^{s}\left(u_{0}\right)=0 \\
l_{0}\left(u_{i}\right)=\lambda_{0} u_{i}+u_{i-1}, \quad U_{0}^{s}\left(u_{i}\right)=0 \\
i=1, \ldots, k-1 ; \quad s=1, \ldots, m .
\end{gathered}
$$


For the adjoint operator $L^{*}$ we have

$$
\begin{gathered}
l_{0}^{*}\left(v_{0}\right)=\overline{\lambda_{0}} v_{0}, \quad V_{0}^{s}\left(v_{0}\right)=0 \\
l_{0}^{*}\left(v_{i}\right)=\overline{\lambda_{0}} v_{i}+v_{i-1}, \quad V_{0}^{s}\left(v_{i}\right)=0 \\
i=1, \ldots, k-1 ; \quad s=1, \ldots, m .
\end{gathered}
$$

The notion of the Keldysh chain is an analogue of the Jordan chain of vectors if we consider the eigenvalue problems for differential operators [1, 5, 7]. Eigenfunctions and associated functions of adjoint operators $L$ and $L^{*}$ are related by the following conditions

$$
\begin{gathered}
\left(u_{j}, v_{0}\right)=0, \quad j=0, \ldots, k-2, \quad\left(u_{k-1}, v_{0}\right) \equiv\left(u_{0}, v_{k-1}\right) \neq 0 \\
\left(u_{j-1}, v_{i}\right) \equiv\left(u_{j}, v_{i-1}\right), \quad i, j,=1, \ldots, k-1 .
\end{gathered}
$$

This naturally follows from equations (12) and (13) subject to the relation $(l(u), v)=\left(u, l^{*}(v)\right)$ stated for the adjoint operators.

The orthogonality of the eigenfunctions $\left(u_{0}, v_{0}\right)=0$ at the points of the flutter boundary of the pure circulatory systems [10] was called "flutter condition" in the work [8]. One can see from (14) that the "flutter condition" is a simple consequence of existing at a multiple eigenvalue the Keldysh chain of length $k \geq 2$.

A variation of the vector of parameters $\mathbf{p}=\mathbf{p}_{0}+\epsilon \mathbf{e}+o(\epsilon)$ leads to the perturbation of eigenvalues and eigenfunctions. In the case of a multiple eigenvalue with the Keldysh chain of length $k$ the expansions of eigenvalues and eigenfunctions contain terms with fractional powers of the small parameter $\epsilon^{j / k}, j=0,1,2, \ldots[6]$ :

$$
\begin{aligned}
& \lambda=\lambda_{0}+\epsilon^{1 / k} \lambda_{1}+\epsilon^{2 / k} \lambda_{2}+\epsilon^{3 / k} \lambda_{3}+\ldots \\
& u=u_{0}+\epsilon^{1 / k} w_{1}+\epsilon^{2 / k} w_{2}+\epsilon^{3 / k} w_{3}+\ldots
\end{aligned}
$$

Substituting expansions (9) and (16) into eigenvalue problem (11), we get expressions which determine the first order perturbations of the eigenvalue $\lambda_{0}$ and the eigenfunction $u_{0}$

$$
\begin{aligned}
& l_{0}\left(w_{j}\right)-\lambda_{0} w_{j}=\lambda_{j} u_{0}+\sum_{i=1}^{j-1>0} \lambda_{j-i} w_{i}=0, \quad U_{0}^{s}\left(w_{j}\right)=0, \quad j=1 \ldots k-1 . \\
& l_{0}\left(w_{k}\right)-\lambda_{0} w_{k}=\lambda_{k} u_{0}-l_{1}\left(u_{0}\right)+\sum_{i=1}^{k-1>0} \lambda_{k-i} w_{i}=0, \quad U_{0}^{s}\left(w_{k}\right)=-U_{1}^{s}\left(u_{0}\right) .
\end{aligned}
$$


The functions $w_{j}$ can be found from equations (12) and (17) in the form

$$
w_{j}=\lambda_{1}^{j} u_{j}+\sum_{p=0}^{j-1} \gamma_{j p} u_{p}, \quad j=1, \ldots, k-1,
$$

where $\gamma_{j p}$ are arbitrary constants.

Consider the inner product of the function $v_{0}$ with the left and right hand sides of (18). Using then expression (19) for $w_{j}$, equations (14), (15)), and the Lagrange identity (8), which in this case has the form

$$
\left(l_{0}\left(w_{k}\right)-\lambda_{0} w_{k}, v_{0}\right)-\left(w_{k}, l_{0}^{*}\left(v_{0}\right)-\overline{\lambda_{0}} v_{0}\right)=-\sum_{s=1}^{m} U_{1}^{s}\left(u_{0}\right) V_{0}^{2 m-s+1}\left(v_{0}\right),
$$

we get the coefficient $\lambda_{1}$ in the expansion of the eigenvalue $\lambda$

$$
\lambda_{1}^{k}=\frac{\left(l_{1}\left(u_{0}\right), v_{0}\right)-\sum_{s=1}^{m} U_{1}^{s}\left(u_{0}\right) V_{0}^{2 m-s+1}\left(v_{0}\right)}{\left(u_{k-1}, v_{0}\right)} .
$$

Introducing the scalar product $\langle\mathbf{a}, \mathbf{b}\rangle$ of vectors $\mathbf{a}, \mathbf{b} \in R^{n}$ and taking into account expressions (10) and (11) we can rewrite (20) in the form [2]

$$
\lambda_{1}=\sqrt[k]{\left\langle\mathbf{f}_{k}, \mathbf{e}\right\rangle+i\left\langle\mathbf{g}_{k}, \mathbf{e}\right\rangle},
$$

where the real vectors $\mathbf{f}_{k}$ and $\mathbf{g}_{k}$ correspond to the $k$ - fold eigenvalue $\lambda_{0}$ at the point $\mathbf{p}=\mathbf{p}_{0}$ and their components are

$$
f_{k}^{j}+i g_{k}^{j}=\frac{\left(\frac{\partial l}{\partial p_{j}}\left(u_{0}\right), v_{0}\right)-\sum_{s=1}^{m} \frac{\partial U^{s}}{\partial p_{j}}\left(u_{0}\right) V_{0}^{2 m-s+1}\left(v_{0}\right)}{\left(u_{k-1}, v_{0}\right)} .
$$

The right hand side of (21) takes $k$ complex values. If the radicand in (21) is not zero, the expression $\lambda=\lambda_{0}+\epsilon^{1 / k} \lambda_{1}+o\left(\epsilon^{1 / k}\right)$ describes the splitting of the $k$ - fold eigenvalue due to change of parameters along a curve emitted in the direction $\mathbf{e}$.

After the splitting the length of the Keldysh chain decreases from $k$ to 1 and we say that the collapse of the Keldysh chain occurs.

In particular, equations (16), (21) describe the behavior of a simple eigenvalue for $k=1$, and for $k=2$ - the splitting of a double eigenvalue $\lambda_{0}$ with the Keldysh chain of length 2 , which are the most important cases in applications.

Consider now for a double eigenvalue the degenerate case

$$
\left\langle\mathbf{f}_{k}, \mathbf{e}\right\rangle+i\left\langle\mathbf{g}_{k}, \mathbf{e}\right\rangle=0 .
$$


It follows from condition (23) that the coefficient $\lambda_{1}$ in expansions (16) becomes zero. Substitution of expansions (16) into eigenvalue problem (1) gives equations determining second-order terms $\lambda_{2}$ and $w_{2}$

$$
\begin{gathered}
l_{0}\left(w_{2}\right)-\lambda_{0} w_{2}=\lambda_{2} u_{0}-l_{1}\left(u_{0}\right), \quad U_{0}^{s}\left(w_{2}\right)=-U_{1}^{s}\left(u_{0}\right), \\
l_{0}\left(w_{4}\right)-\lambda_{0} w_{4}=\lambda_{4} u_{0}-l_{2}\left(u_{0}\right)+\lambda_{2} w_{2}-l_{1}\left(w_{2}\right), \quad U_{0}^{s}\left(w_{4}\right)=-U_{1}^{s}\left(w_{2}\right)-U_{2}^{s}\left(u_{0}\right) .
\end{gathered}
$$

Multiplying both parts of equation (25) by $v_{0}$ and using the Lagrange identity (8) we get

$$
\lambda\left(w_{2}, v_{0}\right)-\left(l_{1}\left(w_{2}\right), v_{0}\right)-\left(l_{2}\left(u_{0}\right), v_{0}\right)+\sum_{s=1}^{m}\left(U_{1}^{s}\left(w_{2}\right)+U_{2}^{s}\left(u_{0}\right)\right) V_{0}^{2 m-s+1}\left(v_{0}\right)=0 .
$$

The similar procedure gives the term $\left(w_{2}, v_{0}\right)$

$$
\left(w_{2}, v_{0}\right)=\lambda_{2}\left(u_{0}, v_{1}\right)-\left(l_{1}\left(u_{0}\right), v_{1}\right)+\sum_{s=1}^{m} U_{1}^{s}\left(u_{0}\right) V_{0}^{2 m-s+1}\left(v_{1}\right) .
$$

The solution of equation (24) has the form $w_{2}=\lambda_{2} u_{1}+\hat{w}_{2}+\gamma u_{0}$, where $\gamma$ is an arbitrary constant and $\hat{w}_{2}$ is a particular solution of the boundary value problem

$$
l_{0}\left(\hat{w}_{2}\right)-\lambda_{0} \hat{w}_{2}=-l_{1}\left(u_{0}\right), \quad U_{0}^{s}\left(\hat{w}_{2}\right)=-U_{1}^{s}\left(u_{0}\right)
$$

The solution of boundary value problem (28) exists due to degeneration condition (23), playing here the role of the solvability condition. Substituting (27) and the expression for $w_{2}$ into (26) we get the quadratic equation on $\lambda_{2}$

$$
\lambda_{2}^{2}+\lambda_{2} a_{1}+a_{2}=0
$$

The coefficients $a_{1}$ and $a_{2}$ are determined by the expressions

$$
a_{1}=\left\langle\mathbf{h}, \mathbf{e}_{*}\right\rangle+i\left\langle\mathbf{k}, \mathbf{e}_{*}\right\rangle, \quad a_{2}=\left\langle\mathbf{H e}_{*}, \mathbf{e}_{*}\right\rangle+i\left\langle\mathbf{K e}_{*}, \mathbf{e}_{*}\right\rangle-\left\langle\mathbf{f}_{2}, \mathbf{d}\right\rangle-i\left\langle\mathbf{g}_{2}, \mathbf{d}\right\rangle .
$$

The real vectors $\mathbf{h}, \mathbf{k}$ and real symmetric matrices $\mathbf{H}, \mathbf{K}$ are defined by the relationships

$$
\begin{gathered}
h^{j}+i k^{j}= \\
\frac{\sum_{s=1}^{m}\left[\frac{\partial U^{s}}{\partial p_{j}}\left(u_{0}\right) V_{0}^{2 m-s+1}\left(v_{1}\right)+\frac{\partial U^{s}}{\partial p_{j}}\left(u_{1}\right) V_{0}^{2 m-s+1}\left(v_{0}\right)\right]}{\left(u_{0}, v_{1}\right)}-\frac{\left(\frac{\partial l}{\partial p_{j}}\left(u_{0}\right), v_{1}\right)+\left(\frac{\partial l}{\partial p_{j}}\left(u_{1}\right), v_{0}\right)}{\left(u_{0}, v_{1}\right)} \\
\frac{H^{j r}+i K^{j r}=}{\left(u_{0}, v_{1}\right)}
\end{gathered}
$$


where the functions $z_{r}(x)\left(\hat{w}_{2}=\sum_{r=1}^{n} e_{*}^{r} z_{r}\right)$ are solutions of the boundary value problems

$$
l_{0}\left(z_{r}\right)-\lambda_{0} z_{r}=-\frac{\partial l}{\partial p_{r}}\left(u_{0}\right), \quad U_{0}^{s}\left(z_{r}\right)=-\frac{\partial U^{s}}{\partial p_{r}}\left(u_{0}\right), \quad r=1 \ldots n .
$$

Thus, bifurcation of the double eigenvalue $\lambda_{0}$ in degenerate case (23) is described by the formula $\lambda=\lambda_{0}+\epsilon \lambda_{2}+o(\epsilon)$, where $\lambda_{2}$ is a root of equation (29).

\section{Application to non-conservative stability problems}

Let us consider the non-conservative systems, described by the partial differential equations

$$
\ddot{y}+l(y)=0, \quad U^{s}(y)=0, \quad s=1 \ldots m
$$

where dots mean differentiation with respect to time $t$, while $l(y), U^{s}(y)$ are respectively the linear differential expression in terms of $x \in[0,1]$ and the boundary forms defined in Section 2. Such systems are usually called circulatory systems [9, 10, 11, 12]. Looking up the solution of equation (33) in the form $y(x, t)=u(x) \exp (i \sqrt{\lambda} t)$ we come to the eigenvalue problem (11). A circulatory system is stable if and only if all the eigenvalues $\lambda$ are positive and semisimple. If all $\lambda$ are real and some of them negative then the circulatory system is statically unstable (divergence). Existence of at least one complex eigenvalue means flutter instability [9, 10, 12].

Recall that the coefficients of the differential expression $l(u)$ and the coefficients of the forms $U^{s}(u)$ are real functions, smoothly dependent on a vector of real parameters $\mathbf{p}=\left(p_{1}, p_{2}, \ldots, p_{n}\right)$. Below we consider only real eigenvalues $\lambda$. It follows from the basic theorems of the theory of ordinary differential equations [14] that solutions $z_{1}, \ldots, z_{m}$ of the equation (1D) with the initial conditions ( $\delta_{j i}$ is the Kronecker symbol)

$$
z_{j}^{(i-1)}(0)=\delta_{j i}, \quad i, j,=1, \ldots, m
$$

forming the fundamental system of solutions of equation (1), smoothly depend on $\lambda$ and $\mathbf{p}$. The characteristic determinant

$$
\Delta \equiv \operatorname{det}\left[\begin{array}{cccc}
U_{1}\left(z_{1}\right) & U_{1}\left(z_{2}\right) & \ldots & U_{1}\left(z_{m}\right) \\
U_{2}\left(z_{1}\right) & U_{2}\left(z_{2}\right) & \ldots & U_{2}\left(z_{m}\right) \\
\vdots & \vdots & \ddots & \vdots \\
U_{m}\left(z_{1}\right) & U_{m}\left(z_{2}\right) & \ldots & U_{m}\left(z_{m}\right)
\end{array}\right]
$$

is thus a smooth function of the spectral parameter $\lambda$ and the vector $\mathbf{p}: \Delta=\Delta(\lambda, \mathbf{p})$. 
We assume that at some fixed value $\mathbf{p}_{0}$ of the vector $\mathbf{p}$ the spectrum of the operator $L$ formed by the differential expression $l(u)$ and boundary conditions $U^{s}(u)=0$ is discrete [5]. The eigenvalues $\lambda$ can be simple or multiple roots of the characteristic equation $\Delta\left(\lambda, \mathbf{p}_{0}\right)=0$. If at $\mathbf{p}=\mathbf{p}_{0}$ the characteristic equation has the $k-$ fold real root $\lambda=\lambda_{0}$, i.e. $\Delta\left(\lambda_{0}, \mathbf{p}_{0}\right)=\partial \Delta / \partial \lambda=\ldots=$ $\partial^{k-1} \Delta / \partial \lambda^{k-1}=0, \partial^{k} \Delta / \partial \lambda^{k} \neq 0$ then according to Malgrange's preparation theorem [15] there exists a neighborhood $U_{0} \subset R \times R^{n}$ of the point $\left(\lambda_{0}, \mathbf{p}_{0}\right)$, where $\Delta(\lambda, \mathbf{p})$ has the form

$$
\Delta(\lambda, \mathbf{p})=\left[\left(\lambda-\lambda_{0}\right)^{k}+\sum_{i=0}^{k-1}\left(\lambda-\lambda_{0}\right)^{i} a_{i}(\mathbf{p})\right] b(\lambda, \mathbf{p}) .
$$

The functions $a_{0}(\mathbf{p}), \ldots, a_{k-1}(\mathbf{p})$ and $b(\lambda, \mathbf{p})$ are smooth, $a_{i}\left(\mathbf{p}_{0}\right)=0, b\left(\lambda_{0}, \mathbf{p}_{0}\right) \neq 0$.

Let for example $\lambda_{0}$ be a simple real root of the equation $\Delta\left(\lambda, \mathbf{p}_{0}\right)=0$. Then, due to (34) we can write $\lambda=\lambda_{0}-a_{0}(\mathbf{p})$, and $\lambda$ remains real and simple in some neighborhood of the point $\mathbf{p}_{0}$. We can conclude from this fact that if at $\mathbf{p}=\mathbf{p}_{0}$ all the eigenvalues of the operator $L$ are positive and simple, then $\mathbf{p}_{0}$ is the inner point of the stability domain of circulatory system (33).

Similarly, the points of the parameter space, corresponding to either simple zero eigenvalue or real double eigenvalue with the Keldysh chain of length 2, form smooth surfaces of dimension $n-1$. Indeed, if $\lambda_{0}=0$ at $\mathbf{p}=\mathbf{p}_{0}$, then in the vicinity of $\mathbf{p}_{0}$ we have $\lambda=-a_{0}(\mathbf{p})$. The equation $a_{0}\left(\mathbf{p}_{0}\right)=0$ defines a hypersurface in the parameter space.

If $\lambda_{0}$ is a double eigenvalue, then according to (34) its behavior near the point $\mathbf{p}_{0}$ is described by the quadratic equation

$$
\left(\lambda-\lambda_{0}\right)^{2}+a_{1}(\mathbf{p})\left(\lambda-\lambda_{0}\right)+a_{0}(\mathbf{p})=0 .
$$

It follows from (35) that the eigenvalue $\lambda(\mathbf{p})$ remains double in the neighborhood of the point $\mathbf{p}_{0}$, if $\mathbf{p}$ belongs to the hypersurface $a_{1}^{2}(\mathbf{p})-4 a_{0}(\mathbf{p})=0$.

It is clear that the stability of the system in the vicinity of these hypersurfaces depends on behavior of the zero or the double eigenvalues due to change of parameters. According to (16), (21), where we should put $k=1$ or $k=2$, the behavior of the simple zero eigenvalue is described by the formula

$$
\lambda=\epsilon\left\langle\mathbf{f}_{1}, \mathbf{e}\right\rangle+o(\epsilon),
$$

and the splitting of the real double $\lambda_{0}$ is governed by the expression

$$
\lambda=\lambda_{0} \pm \sqrt{\epsilon\left\langle\mathbf{f}_{2}, \mathbf{e}\right\rangle}+o\left(\epsilon^{1 / 2}\right) .
$$


The inequality $\left\langle\mathbf{f}_{1}, \mathbf{e}\right\rangle>0$ defines a set of such directions $\mathbf{e}$ that the curves $\mathbf{p}=\mathbf{p}(\epsilon)$ emitted along these vectors lie in the stability domain, i.e. a tangent cone to the stability domain. The eigenvalue $\lambda$ becomes negative at $\left\langle\mathbf{f}_{1}, \mathbf{e}\right\rangle<0$. Consequently, this inequality gives a tangent cone to the static instability (divergence) domain. The eigenvalue remains zero up to the terms of order $\epsilon^{2}$ on the curves, emitted in the directions $\mathbf{e}$, such that $\left\langle\mathbf{f}_{1}, \mathbf{e}\right\rangle=0$. Thus, the equation $\left\langle\mathbf{f}_{1}, \mathbf{p}-\mathbf{p}_{0}\right\rangle=0$ defines a tangent plane to the surface, where the operator $L$ has a simple zero eigenvalue. If other eigenvalues remain simple and positive on this surface, then it forms a boundary between stability and divergence domains. The vector $\mathbf{f}_{1}$ is the normal vector to the boundary and is directed to the stability domain.

Analyzing splitting of the double eigenvalue with the formula (37) we can show that the points of the parameter plane, corresponding to the real double eigenvalue with the Keldysh chain of length 2 , belong to the smooth parts of the boundary between the flutter domain and the stability domain if $\lambda_{0}>0$, or divergence domain if $\lambda_{0}<0$. In this case the vector $\mathbf{f}_{2}$ is the normal vector to the flutter boundary looking at the stability or divergence domains, respectively.

\section{Stability boundaries of the extended Beck problem}

As an example of a continuous non-conservative mechanical system we first consider a uniform elastic cantilevered column of length $L_{c}$, Fig. 1. Assume that the non-conservative force $P$, which can be represented as the sum of a tangential follower force and a potential load, is acting at the free end of the column. The parameter $\eta \in[0,1]$ measures the non-conservativity of the force $P$. The case $\eta=1$ means that the column is loaded by purely tangential follower force (Beck's problem [16]). If $\eta=0$, then the force $P$ is potential (conservative).

Consider the transverse vibrations of the column in the plane $O X Y$, Fig. 1. In the nondimensional variables

$$
x=X / L_{c}, \quad y=Y / L_{c}, \quad \tau=t / \sqrt{\rho A L_{c}^{4} / E I}, \quad q=P L_{c}^{2} / E I
$$

the differential equation describing small in-plane vibrations of the column and the appropriate boundary conditions have the form

$$
\begin{gathered}
y^{\prime \prime \prime \prime}(x, \tau)+q y^{\prime \prime}(x, \tau)+\ddot{y}(x, \tau)=0, \\
u(0, \tau)=u^{\prime}(0, \tau)=u^{\prime \prime}(1, \tau)=u^{\prime \prime \prime}(1, \tau)+(1-\eta) q u^{\prime}(1, \tau)=0 .
\end{gathered}
$$


Dots mean differentiation with respect to time $\tau$ and primes denote differentiation w.r.t. coordinate $x$.

Separating time by $y(x, \tau)=u(x) \exp (i \sqrt{\lambda} \tau)$, we get the eigenvalue problem [17, [18]

$$
\begin{gathered}
l(u) \equiv u^{\prime \prime \prime \prime}+q u^{\prime \prime}=\lambda u \\
U^{1}(u) \equiv u(0)=0, \\
U^{2}(u) \equiv u^{\prime}(0)=0, \quad U^{4}(u) \equiv u^{\prime \prime}(1)=0, \\
u^{\prime \prime \prime}(1)+(1-\eta) q u^{\prime}(1)=0 .
\end{gathered}
$$

The corresponding adjoint eigenvalue problem looks like

$$
\begin{gathered}
l^{*}(v) \equiv v^{\prime \prime \prime \prime}+q v^{\prime \prime}=\lambda v \\
V^{1}(v) \equiv-v(0)=0, \quad V^{3}(v) \equiv v^{\prime \prime}(1)+\eta q v(1)=0, \\
V^{2}(v) \equiv v^{\prime}(0)=0, \quad V^{4}(v) \equiv-v^{\prime \prime \prime}(1)-q v^{\prime}(1)=0,
\end{gathered}
$$

and for the forms $V^{5} \ldots V^{8}$ we have

$$
V^{5} \equiv v(1), \quad V^{7} \equiv-v^{\prime \prime}(0)-q v(0), \quad V^{6} \equiv-v^{\prime}(1), \quad V^{8} \equiv v^{\prime \prime \prime}(0)+q v^{\prime}(0) .
$$

Substituting the general solution of differential equation (38)

$$
\begin{aligned}
& u(x)=C_{1} \cosh (a x)+C_{2} \sinh (a x)+C_{3} \cos (b x)+C_{4} \sin (b x), \\
& a=\sqrt{-\frac{q}{2}+\sqrt{\frac{q^{2}}{4}+\lambda}}, \quad b=\sqrt{\frac{q}{2}+\sqrt{\frac{q^{2}}{4}+\lambda}}, \quad \lambda \neq-\frac{q^{2}}{4} .
\end{aligned}
$$

into boundary conditions (39) we obtain the condition of existing of a non-trivial solution $u(x)$ to eigenvalue problem (38), (39) in the form [18]

$$
\begin{gathered}
\Delta(\lambda, \eta, q)=0, \\
\Delta \equiv\left(2 \lambda+(1-\eta) q^{2}\right)(1+\cosh (a) \cos (b))+q(2 \eta-1)(q+a b \sinh (a) \sin (b)) .
\end{gathered}
$$

Equation (43) gives eigenvalues $\lambda$, depending on the parameters $\eta$ and $q$.

The vertical equilibrium of the column is stable if all the eigenvalues $\lambda$ are positive and semisimple, i.e. each eigenvalue has the same number of eigenfunctions as its algebraic multiplicity. After substitution $\lambda=0$ in equation (43) it gives possible values of the parameters $\eta$ and $q$ at which the system loses stability statically [17]

$$
\eta(q)=\frac{\cos (\sqrt{q})}{\cos (\sqrt{q})-1} .
$$



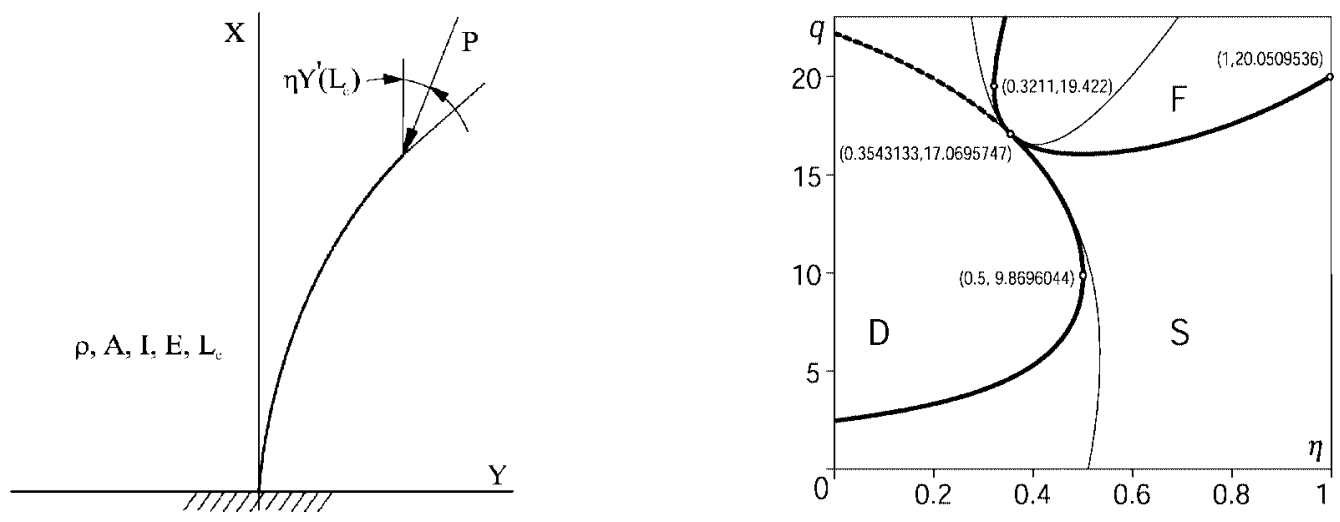

Figure 1: Extended Beck's problem and its stability diagram.

Equation (44) defines the curve of simple zero eigenvalues, the part of which forms the boundary between stability and divergence domains on the plane of parameters $(\eta, q)$. The smooth parts of the flutter boundary consist of such points $(\eta, q)$ that $\lambda(\eta, q)$ is a double real eigenvalue. Calculation of the roots of characteristic equation (43) for the different values of the load parameter $q$ (at a fixed value of the parameter $\eta$ ) gives approximately the point, where two simple eigenvalues form a double. Finding such points for the different values of the parameter $\eta$ we get the curve of double real eigenvalues.

The curves found subdivide the plane of the parameters $(\eta, q)$ into stability (S), flutter (F), and divergence (D) domains, Fig. 1. The boundaries between these domains are shown on Fig. 1. by the firm thick lines while the dashed thick line shows the part of the curve of zero eigenvalues, which belongs to the divergence domain. Note that the boundary of stability domain has a singular point where the smoothness of the boundary is broken. The divergence boundary has two points with the vertical tangents. One can see that while the influence of the non-conservative part of the load $q$ is small $(\eta<0.5)$ the column loses stability by divergence. Mainly non-conservative load $(\eta>0.5)$ causes dynamical instability.

Our goal here is to demonstrate on the example of Beck's problem the advantages of the theory developed in the previous sections. It will be applied to finding linear and quadratic approximations of the stability and instability domains both at singular and regular points of their boundaries. The explicit expression describing the overlapping of the frequency curves near the flutter boundary will be obtained and compared with the numerical results, described in [18]. Finally, we will obtain the exact coordinates of the singular point of the stability boundary, show that this point corresponds to the double zero eigenvalue with the Keldysh chain of length 2, and investigate the splitting of 
this eigenvalue in the vicinity of the singularity.

\section{Bifurcation of eigenvalues in the vicinity of the flutter boundary.}

Consider a point $\mathbf{p}_{0}=\left(\eta_{0}, q_{0}\right)$ of the flutter boundary, where the spectrum of the operator $L$ contains double eigenvalue $\lambda_{0}$ with the Keldysh chain of length 2. Bifurcation of this eigenvalue is described by equation (37). Substituting the differential expression $l(u)$ from (38), the forms $U^{1}, \ldots, U^{4}$ and $V^{5}, \ldots, V^{8}$ from (39), (42) into formula (22), we get the normal vector to the boundary

$$
\mathbf{f}_{2}=\left(\frac{q_{0} u_{0}^{\prime}(1) v_{0}(1)}{\int_{0}^{1} u_{0} v_{1} d x}, \frac{\int_{0}^{1} u_{0}^{\prime \prime} v_{0} d x-\left(1-\eta_{0}\right) u_{0}^{\prime}(1) v_{0}(1)}{\int_{0}^{1} u_{0} v_{1} d x}\right) .
$$

For evaluation of the vector $\mathbf{f}_{2}$ it is essential to know the eigenfunctions $u_{0}, v_{0}$ as well as the associated functions $u_{1}, v_{1}$ at the double eigenvalue $\lambda_{0}$. Solution of eigenvalue problems (38), (39) and (40), (41) yields [19]

$$
\begin{aligned}
& u_{0}(x)=\cosh (a x)-\cos (b x)+F(a \sin (b x)-b \sinh (a x)), \\
& v_{0}(x)=\cosh (a x)-\cos (b x)+G(a \sin (b x)-b \sinh (a x)),
\end{aligned}
$$

where the coefficients $F$ and $G$ depend on the parameters $\eta$ and $q$

$$
F=\frac{a^{2} \cosh (a)+b^{2} \cos (b)}{a b(a \sinh (a)+b \sin (b))}, \quad G=\frac{\left.\left(a^{2}+\eta q\right) \cosh (a)+\left(b^{2}-\eta q\right) \cos (b)\right)}{b\left(a^{2}+\eta q\right) \sinh (a)+a\left(b^{2}-\eta q\right) \sin (b)}
$$

Associated function $u_{1}$ is a particular solution of the boundary value problem (12), where we should put $k=2$ and take the differential expression and the boundary forms from (38), (39). A particular solution of ordinary linear differential equation with constant coefficients

$$
u_{1}^{\prime \prime \prime \prime}+q u_{1}^{\prime \prime}-\lambda_{0} u_{1}=u_{0}
$$

whose right hand side is the linear combination of trigonometric and hyperbolic functions (46), has the form

$$
\hat{u}_{1}=x\left(C_{1} \sin (b x)+C_{2} \cos (b x)+C_{3} \sinh (a x)+C_{4} \cosh (a x)\right) .
$$

Substitution of $\hat{u}_{1}$ into equation (12) allows to determine the coefficients $C_{1}, \ldots, C_{4}$. After these coefficients are found one tries solution of boundary value problem (12) in the form

$$
u_{1}=\hat{u}_{1}+D_{1} \sin (b x)+D_{2} \cos (b x)+D_{3} \sinh (a x)+D_{4} \cosh (a x) .
$$


The boundary conditions (12) serve for obtaining the unknown constants $D_{1}, \ldots, D_{4}$. After all necessary manipulations we arrive at the associated function $u_{1}$

$$
\begin{gathered}
u_{1}(x)=\frac{a \sin (b x)+b \sinh (a x)+F\left(a^{2} \cos (b x)-b^{2} \cosh (a x)\right)}{2 a b\left(a^{2}+b^{2}\right)} x+ \\
+\frac{A_{1} \sinh (a x)-B_{1} \sin (b x)}{2 a b\left(a^{2}+b^{2}\right)(a \sinh (a)+b \sin (b))^{2}},
\end{gathered}
$$

where the coefficient $F$ is taken from (48) while for the coefficients $A_{1}, B_{1}$ we have

$$
\begin{gathered}
A_{1}=\frac{\sin (b)\left(b^{2} \cos (b)-a^{2} \cosh (a)\right)+2 a b \cos (b) \sinh (a)}{a^{2}} q+ \\
+b\left(a^{2}+b^{2}\right)(1+\cosh (a) \cos (b)), \\
B_{1}=\frac{\sinh (a)\left(b^{2} \cos (b)-a^{2} \cosh (a)\right)-2 a b \cosh (a) \sin (b)}{b^{2}} q+ \\
+a\left(a^{2}+b^{2}\right)(1+\cosh (a) \cos (b)) .
\end{gathered}
$$

Similarly, solving boundary value problem (13) with the differential expression and boundary forms from (40), (41) we get the associated function $v_{1}$

$$
\begin{aligned}
v_{1}(x) & =\frac{a \sin (b x)+b \sinh (a x)+G\left(a^{2} \cos (b x)-b^{2} \cosh (a x)\right)}{2 a b\left(a^{2}+b^{2}\right)} x+ \\
+ & \frac{A_{2} \sinh (a x)-B_{2} \sin (b x)}{2 a b\left(a^{2}+b^{2}\right)\left(b\left(a^{2}+\eta q\right) \sinh (a)+a\left(b^{2}-\eta q\right) \sin (b)\right)^{2}},
\end{aligned}
$$

where the coefficient $G$ is defined in (48) and the coefficients $A_{2}, B_{2}$ are

$$
\begin{aligned}
A_{2}= & q \sin (b)\left[\left[-a^{2} b^{2}+\eta\left(\left(a^{2}+b^{2}\right)^{2}-\eta q^{2}\right)\right] \cosh (a)+\cos (b)\left(b^{2}-\eta q\right)^{2}\right]+ \\
& +2 q a b^{3}(1-2 \eta) \sinh (a) \cos (b)+ \\
& +b\left(a^{2}+b^{2}\right)\left[a^{2} b^{2}+\eta^{2} q^{2}+\left(a^{2} b^{2}+\eta(1-\eta) q^{2}\right) \cos (b) \cosh (a)\right] ; \\
B_{2}= & q \sinh (a)\left[\left[a^{2} b^{2}-\eta\left(\left(a^{2}+b^{2}\right)^{2}-\eta q^{2}\right)\right] \cos (b)-\cosh (a)\left(a^{2}+\eta q\right)^{2}\right]- \\
& -2 q b a^{3}(1-2 \eta) \sin (b) \cosh (a)+ \\
& +a\left(a^{2}+b^{2}\right)\left[a^{2} b^{2}+\eta^{2} q^{2}+\left(a^{2} b^{2}+\eta(1-\eta) q^{2}\right) \cos (b) \cosh (a)\right] .
\end{aligned}
$$

The possibility of existing of associated functions at the critical values of the non-conservative load in Beck's problem was noted earlier in the work [20]. Nevertheless the explicit expressions 

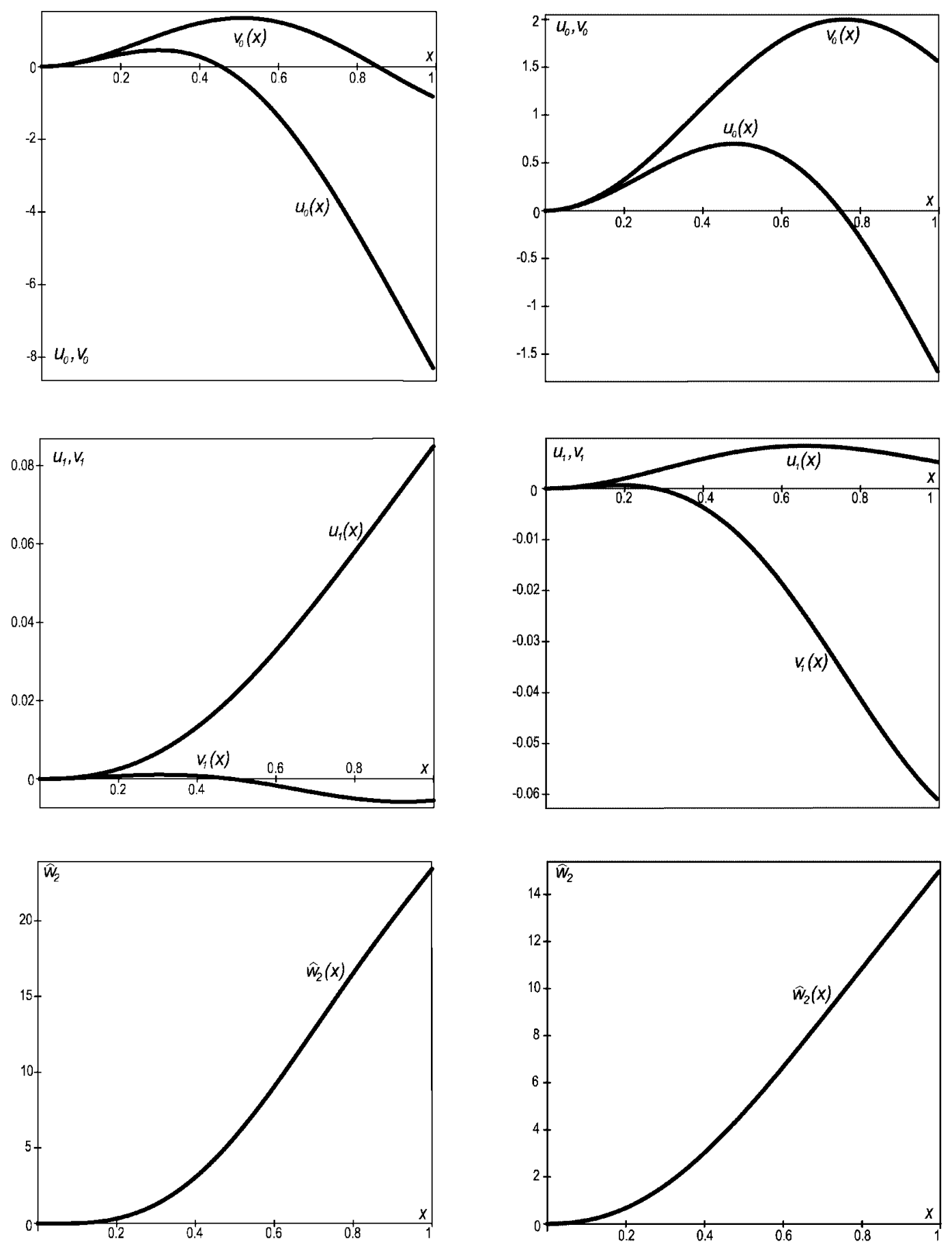

Figure 2: The eigenfunctions, associated functions and functions $\hat{w}_{2}(x)$ at the points (from left to right $)(1,20.0509536)$ and $(0.35431330,17.0695748)$. 
for the associated functions in Beck's problem seem to be obtained first in the present paper. Note that despite of the eigenfunctions $u_{0}, v_{0}$ are defined to within arbitrary multipliers and associated functions $u_{1}, v_{1}$ are defined to within the addends $C_{1} u_{0}$ and $C_{2} v_{0}$, respectively, the vector $\mathbf{f}_{2}$ does not depend on these uncertainties.

Consider now the point $\mathbf{p}_{0}=(1,20.0509536)$, corresponding to the double eigenvalue $\lambda_{0}=$ 121.347049. This point is known as critical for the column subjected to a pure tangential follower force [16]. Substituting the values of $\lambda_{0}$ and $\mathbf{p}_{0}$ into (46) $-(50)$ we obtain the functions $u_{0}, v_{0}$, $u_{1}, v_{1}$, Fig 2 . With the use of these functions equation (45) gives the normal vector to the flutter boundary at the point $\mathbf{p}_{0}=(1,20.0509536)$

$$
\mathbf{f}_{2}=(35458.3181,-2296.10536) \text {. }
$$

Let us look at the splitting of the double eigenvalue $\lambda_{0}$ due to change of parameters in different directions $\mathbf{e}$ on the parameter plane. Consider for example the vertical direction: $\mathbf{e}=(0,1)$. Taking into account that $\Delta \mathbf{p}=\epsilon \mathbf{e}=\left(0, q-q_{0}\right)$ we get from (37)

$$
\lambda=121.347049 \pm 47.9176936 \sqrt{q_{0}-q} .
$$

For the horizontal variation $\Delta \mathbf{p}=\left(\eta-\eta_{0}, 0\right)$ corresponding to the vector $\mathbf{e}=(1,0)$ we have

$$
\lambda=121.347049 \pm 188.303792 \sqrt{\eta-\eta_{0}} .
$$

The results of probing of a small neighborhood of the point $\mathbf{p}_{0}$ in different directions are summarized in Tab. 1. Thus, for example, for $q=q_{0}+0.00002$, i.e. when the new point is situated above the initial point $\mathbf{p}_{0}$, splitting yields $\lambda=121.347049 \pm i 0.21429444$, and thus the point $\mathbf{p}_{0}+\Delta \mathbf{p}$ belongs to the flutter domain, Fig. 1. Characteristic equation (43) gives for the same values of parameters two complex-conjugate eigenvalues, which differ from those found with the use of (51) only in a sixth digit, Tab. 1.

Degeneration condition (23) defines the vector $\mathbf{e}_{*}=(-1,-15.4428097)$ tangent to the flutter boundary at the point $\mathbf{p}_{0}=(1,20.0509536)$. Double eigenvalue $\lambda_{0}$ splits in the tangent direction in accordance with equation (29)

$$
\lambda=\lambda_{0}-\frac{a_{1}}{2} \epsilon \pm \frac{\epsilon}{2} \sqrt{a_{1}^{2}-4 a_{2}}+o(\epsilon) .
$$


Table 1: Splitting of the double eigenvalue near the point $\mathbf{p}_{0}=(1,20.0509536)$.

\begin{tabular}{cll}
\hline$(\Delta \eta, \Delta q)$ & $\lambda:$ Eqs.(51),(52),(56). & $\lambda: E q .(43)$ \\
\hline$\left(0,2 \cdot 10^{-5}\right)$ & $\operatorname{Re} \lambda_{1,2}=121.347049$ & $\operatorname{Re} \lambda_{1,2}=121.342379$ \\
& $\operatorname{Im} \lambda_{1,2}= \pm 0.21429444$ & $\operatorname{Im} \lambda_{1,2}= \pm 0.21422599$ \\
$\left(0,-2 \cdot 10^{-5}\right)$ & $\lambda_{1}=121.132755$ & $\lambda_{1}=121.128319$ \\
& $\lambda_{2}=121.561343$ & $\lambda_{2}=121.556963$ \\
$\left(2 \cdot 10^{-5}, 0\right)$ & $\lambda_{1}=122.189169$ & $\lambda_{1}=122.188528$ \\
& $\lambda_{2}=120.504929$ & $\lambda_{2}=120.504432$ \\
$\left(-2 \cdot 10^{-5}, 0\right)$ & $\operatorname{Re} \lambda_{1,2}=121.347049$ & $\operatorname{Re} \lambda_{1,2}=121.338540$ \\
& $\operatorname{Im} \lambda_{1,2}= \pm 0.84212016$ & $\operatorname{Im} \lambda_{1,2}= \pm 0.84201129$ \\
$2 \cdot 10^{-3} \mathbf{e}_{*}$ & $\lambda_{1}=120.762103$ & $\lambda_{1}=120.755389$ \\
& $\lambda_{2}=121.542851$ & $\lambda_{2}=121.540824$ \\
\hline
\end{tabular}

Substitution of the differential expression $l(u)$ from (38), and the forms $U^{1}, \ldots, U^{4}, V^{5}, \ldots, V^{8}$ from (39) and (42) into (30) - (32) gives the coefficients $a_{1}$ and $a_{2}$ in the form

$$
\begin{gathered}
a_{1}=\frac{e_{2}^{*} \int_{0}^{1}\left(u_{0}^{\prime} v_{1}^{\prime}+v_{0}^{\prime} u_{1}^{\prime}\right) d x-\left(e_{2}^{*} \eta_{0}+e_{1}^{*} q_{0}\right)\left(v_{1}(1) u_{0}^{\prime}(1)+v_{0}(1) u_{1}^{\prime}(1)\right)}{\int_{0}^{1} u_{0} v_{1} d x}, \\
a_{2}=\frac{e_{2}^{*} \int_{0}^{1} v_{0}^{\prime} \hat{w}_{2}^{\prime} d x-\left(e_{2}^{*} \eta_{0}+e_{1}^{*} q_{0}\right) v_{0}(1) \hat{w}_{2}^{\prime}(1)-e_{1}^{*} e_{2}^{*} v_{0}(1) u_{0}^{\prime}(1)}{\int_{0}^{1} u_{0} v_{1} d x} .
\end{gathered}
$$

The functions $u_{0}, v_{0}, u_{1}, v_{1}$ are presented by equations (46), (47), (49), (50). The function $\hat{w}_{2}(x)$, Fig. 2., is a solution of boundary value problem (28), where the differential expressions $l_{0}, l_{1}$ and forms $U_{0}^{s}, U_{1}^{s}$ are derived from differential expression (38) and boundary forms (39) according to (10), (11)

$$
\begin{gathered}
\hat{w}_{2}(x)=\frac{b \sin (b x)-a \sinh (a x)+F a b(\cos (b x)+\cosh (a x))}{2\left(a^{2}+b^{2}\right)} e_{2}^{*} x+ \\
+\frac{A_{3} \sin (b x)-B_{3} \sinh (a x)}{2 a b\left(a^{2}+b^{2}\right)(b \sin (b)+a \sinh (a))^{2}} e_{2}^{*} .
\end{gathered}
$$

The coefficient $F$ in (55) is defined in (48) and for the coefficients $A_{3}$ and $B_{3}$ we have

$$
A_{3}=-a\left(a^{2}+b^{2}\right)(q+a b \sin (b) \sinh (a))+2 a^{2} b(b \sinh (a) \cos (b)-a \cosh (a) \sin (b))-
$$




$$
\begin{gathered}
-2 a^{3} \cosh (a)(a \sinh (a)+b \sin (b)), \\
B_{3}=-b\left(a^{2}+b^{2}\right)(q+a b \sin (b) \sinh (a))+2 b^{2} a(b \sinh (a) \cos (b)-a \cosh (a) \sin (b))+ \\
+2 b^{3} \cos (b)(a \sinh (a)+b \sin (b)) .
\end{gathered}
$$

With the use of the eigenfunctions, associated functions and function $\hat{w}_{2}$ we find from equations (54) the coefficients $a_{1}=194.571965, a_{2}=-28633.4466$. Substitution of these coefficients into equation (53) gives approximate expressions for two simple eigenvalues which result from the splitting of the double $\lambda_{0}$ in the tangent direction to the stability boundary

$$
\lambda_{1}=121.347049-292.473089 \epsilon, \quad \lambda_{2}=121.347049+97.9011324 \epsilon .
$$

Let for example take $\epsilon=0.002$, then the double eigenvalue $\lambda_{0}$ splits into two positive eigenvalues, Tab. 1. This means that the tangent vector $\mathbf{e}_{*}=(-1,-15.4428097)$ lies in the stability domain whence it follows that the flutter domain is convex at the point $\mathbf{p}_{0}$, Fig.1. At the same values of the parameters the characteristic equation has very close solutions, Tab. 1, showing thereby that the perturbation theory formulae (56) give a good approximation to the directly computed eigenvalues.

Consider now the point $\mathbf{p}_{0}=(0.32112653,19.4220703)$ on the boundary between flutter and divergence domains, Fig. 1. In this point there exists the negative double eigenvalue $\lambda_{0}=$ -46.4046486 with Keldysh chain of length 2 . The normal vector to the flutter boundary evaluated at this point by formula (45) is

$$
\mathbf{f}_{2}=(-53123.691,0)
$$

The corresponding tangent vector to the boundary follows from degeneration condition (23)

$$
\mathbf{e}_{*}=(0,1)
$$

One can see that the normal vector is parallel to the $\eta$-axis and is situated in the divergence domain, so the flutter boundary has a vertical tangent at the point $\mathbf{p}_{0}=(0.32112653,19.4220703)$, Fig. 1.

We are interested now in behavior of the frequency curves $\omega(q)$, where $\omega=\sqrt{\lambda}$ is a frequency of oscillations, in the vicinity of the point $\mathbf{p}_{0}$ of the boundary between the flutter and divergence 

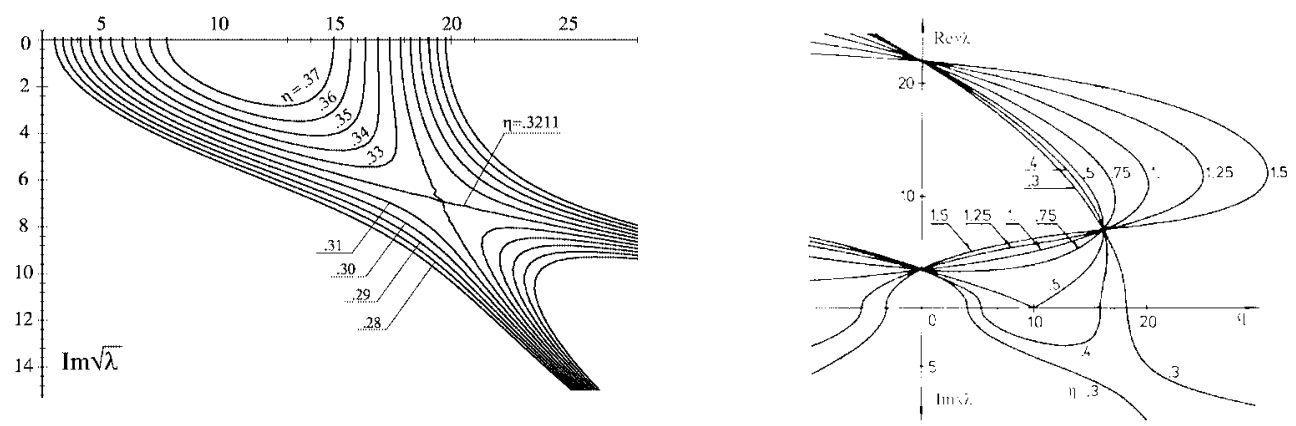

Figure 3: The frequency curves (from left to right) by Eq. (58) and by the work [18].

domains. From the formula (29) it follows that along the curves $\mathbf{p}=\mathbf{p}_{0}+\epsilon \mathbf{e}_{*}+\epsilon^{2} \mathbf{d}+o\left(\epsilon^{2}\right)$ the double eigenvalue splits according to equation [21]

$$
\left(\lambda-\lambda_{0}\right)^{2}+\left\langle\mathbf{h}, \mathbf{e}_{*}\right\rangle\left(\lambda-\lambda_{0}\right) \epsilon+\left\langle\mathbf{H e}_{*}, \mathbf{e}_{*}\right\rangle \epsilon^{2}=\epsilon^{2}\left\langle\mathbf{f}_{2}, \mathbf{d}\right\rangle+o\left(\epsilon^{2}\right) .
$$

Taking into account that along the curve $\mathbf{p}(\epsilon)$ tangent to the flutter boundary at the point $\mathbf{p}_{0}$

$$
q-q_{0}=\epsilon e_{*}^{2}+o(\epsilon), \quad \eta-\eta_{0}=\epsilon e_{*}^{1}+\epsilon^{2} d^{1}+o\left(\epsilon^{2}\right)
$$

we convert expression (57) into

$$
\left(\lambda-\lambda_{0}+\frac{h^{2}}{2}\left(q-q_{0}\right)\right)^{2}-\left(\frac{\left(h^{2}\right)^{2}}{4}-H^{22}\right)\left(q-q_{0}\right)^{2}=f_{2}^{1}\left(\eta-\eta_{0}\right) .
$$

Formulae (31), (32) give the components of the vector $\mathbf{h}$ and matrix $\mathbf{H}$

$$
\begin{gathered}
h^{1}=686267882692882 ., \quad h^{2}=32.1039479, \\
H^{11}=0, \quad H^{12}=1917.18297, \quad H^{22}=93.4817323 .
\end{gathered}
$$

The double eigenvalue $\lambda_{0}$ does not split in the first approximation if the discriminant of (58) is zero. This condition gives us the quadratic approximation of the flutter boundary near the point $\mathbf{p}_{0}=(0.32112653,19.4220703)$

$$
\eta=0.32112653+0.0030906(q-19.4220703)^{2} .
$$

Equation (59) shows that the flutter domain is convex at the point $\mathbf{p}_{0}$, see Fig. 1. Equation (58) approximates in the vicinity of the point $q_{0}, \lambda_{0}$ the family of frequency curves $\omega(q)=\sqrt{\lambda(q)}$, 
parameterized by $\eta$, if we assume $\lambda=-(\operatorname{Im} \sqrt{\lambda})^{2}$. At $\eta=\eta_{0}$ equation (58) disintegrates into two parts

$$
q_{1}=0.30878129(\operatorname{Im} \sqrt{\lambda})^{2}+5.09318321, \quad q_{2}=0.03464354(\operatorname{Im} \sqrt{\lambda})^{2}+17.814449 .
$$

Parabolae (60) are symmetrical with respect to the axis $q$ and are situated on the plane $(q, \operatorname{Im} \sqrt{\lambda})$. At the points $(19.4220703, \pm 6.81209576)$ corresponding to two purely imaginary eigenfrequencies $\omega= \pm i 6.81209576$ these parabolae intersect.

On the left picture on Fig. 3 behavior of frequency curves described by equation (58) near one of the intersecting points is shown. One can see that at $\eta<\eta_{0}$ there exist two purely imaginary frequencies meaning the static instability. With the increase of $\eta$ frequency curves come closer together, overlap and at $\eta>\eta_{0}$ move apart, forming a zone of complex eigenvalues (flutter). On the right picture on Fig. 3 the dependence of the two lowest eigenfrequencies $\omega=\sqrt{\lambda}$ on the load $q$ at the different values of parameter $\eta \in[0.3,1.5]$, obtained earlier in the work [18] by numerical solution of characteristic equation (43) is shown. Comparing two pictures on Fig. 3 note a good qualitative and quantitative agreement in behavior of frequency curves calculated by two different methods on the range $\eta \in[0.3,0.4]$.

\section{Behavior of eigenvalues near the boundary between divergence and stability domains.}

Consider a point $\mathbf{p}_{0}=\left(\eta_{0}, q_{0}\right)$ on the boundary between the stability and divergence domains, where the spectrum of the eigenvalue problem (38), (39) contains a simple eigenvalue $\lambda_{0}=0$. Due to variation of parameters a simple eigenvalue changes according to formula (36). Substituting the differential expression $l(u)$ from (苂8), the forms $U^{1}, \ldots, U^{4}$ and $V^{5}, \ldots, V^{8}$ from (39), (42) into (22) we get the normal vector $\mathbf{f}_{1}$

$$
\mathbf{f}_{1}=\left(\frac{q_{0} u_{0}^{\prime}(1) v_{0}(1)}{\int_{0}^{1} u_{0} v_{0} d x}, \frac{\int_{0}^{1} u_{0}^{\prime \prime} v_{0} d x-\left(1-\eta_{0}\right) u_{0}^{\prime}(1) v_{0}(1)}{\int_{0}^{1} u_{0} v_{0} d x}\right)
$$

The eigenfunctions $u_{0}$ and $v_{0}$ at the simple zero eigenvalue have the form

$$
\begin{gathered}
u_{0}=\sin (b)-x b \cos (b)-\sin (b) \cos (b x)+\cos (b) \sin (b x), \\
v_{0}=1-\cos (b x), \quad b=\sqrt{q_{0}} .
\end{gathered}
$$

These eigenfunctions are solutions of eigenvalue problems $(38)-(41)$ at $\lambda_{0}=0$, and are presented on Fig. 4. 


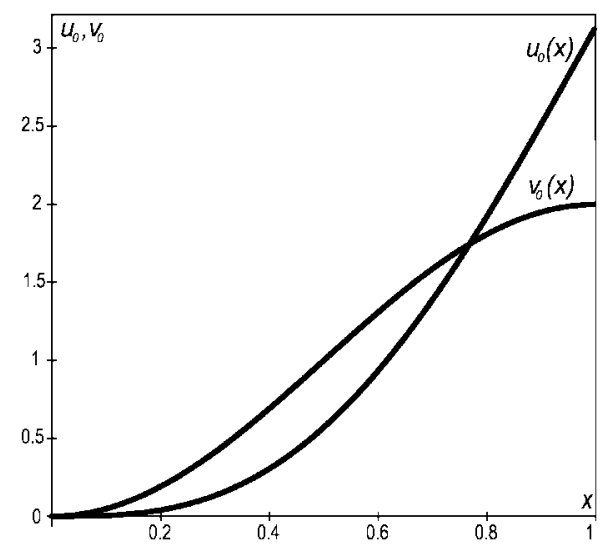

Figure 4: The eigenfunctions of the zero eigenvalue at the point $\mathbf{p}_{0}=(0.5,9.86960440)$.

Consider the point $\mathbf{p}_{0}=(0.5,9.86960440)$ on the divergence boundary described by equation (44). Substituting eigenfunctions (62), (63) evaluated at this point into (61) we get the normal vector to the divergence boundary

$$
\mathbf{f}_{1}=(78.9568352,0)
$$

Hence, the divergence boundary has the vertical tangent at the point $\mathbf{p}_{0}$, Fig. 1. Variation of parameters $\Delta \mathbf{p}=\left(\eta-\eta_{0}, 0\right)$ changes the zero eigenvalue. According to (36) we have

$$
\lambda=78.956835\left(\eta-\eta_{0}\right) .
$$

One can see that for $\eta-\eta_{0}<0$ the eigenvalue $\lambda_{0}=0$ becomes negative. Therefore, the point $\mathbf{p}_{0}+\Delta \mathbf{p}$ is inside the divergence domain, Fig. 1 . If $\eta-\eta_{0}>0$ we come to the stability domain, Tab. 2.

The singularity $0^{2}$ of the stability boundary. Figure 1 clearly shows that the flutter domain has a common boundary with domains of stability and divergence. Recall that at the points of the boundary between flutter and stability domains the spectrum of the differential operator contains positive double eigenvalues while at the points of the boundary between the flutter and divergence domains double eigenvalues are negative.

Thus, the double eigenvalue becomes double zero at such point of the flutter boundary that separates stability and divergence domains. At the same time the point with double zero eigenvalue should belong to the curve of zero eigenvalues (44)). Besides, due to (14) the orthogonality condition $\int_{0}^{1} u_{0} v_{0} d x=0$ must be true at the points of the flutter boundary. It is clear that this integral evaluated at the points of curve (44) becomes zero only at the point, corresponding to the double zero eigenvalue. 
Table 2: Changing of zero eigenvalue near the point $\mathbf{p}_{0}=(0.5,9.86960440)$.

\begin{tabular}{lll}
\hline$(\Delta \eta, \Delta q)$ & $\lambda: E q \cdot(64)$ & $\lambda: E q \cdot(43)$ \\
\hline$\left(10^{-4}, 0\right)$ & $\lambda=0.00789568$ & $\lambda=0.00789498$ \\
$\left(-10^{-4}, 0\right)$ & $\lambda=-0.00789568$ & $\lambda=-0.00789639$ \\
\hline
\end{tabular}

Integrating the product of the eigenfunctions $u_{0}(x)$ and $v_{0}(x)$ from $(\underline{62})$, (63) over the range $[0,1]$ we come to the transcendental equation, which the ordinate of the desired point must satisfy

$$
q_{0}=\left(\sqrt{q_{0}}-2 \sin \left(\sqrt{q_{0}}\right)\right)\left(\sqrt{q_{0}}\left(1+2 \cos \left(\sqrt{q_{0}}\right)\right)-4 \sin \left(\sqrt{q_{0}}\right)\right) .
$$

The minimal element of the set of solutions of equation ([65) at $q_{0}>0$ is $q_{0}=17.0695748$. Substituting this solution into equation (44), we find the corresponding value of the second parameter $\eta_{0}=0.35431330$.

Note that equation similar to (65) was derived first in the work [17] from the analysis of characteristic equation (43) and without use of the eigenfunctions. Unfortunately, formula (3.23) of the article [17] contains a misprint: the first term $k_{2}^{2} l^{2} \cos ^{2} k_{2} l$ should be read as $k_{2}^{2} l^{2} \cos k_{2} l$. Nevertheless, the coordinates of the singular point found in [17] are correct and coincide with those obtained from equation (65]).

Thus, at the point $\mathbf{p}_{0}=(0.35431330,17.0695748)$ there exists the double eigenvalue $\lambda_{0}=0$ with Keldysh chain of length 2. Following V.I. Arnold [22] we denote this point by the symbol $0^{2}$, where the upper index means the length of the Keldysh chain corresponding to the double zero eigenvalue.

The bifurcation of double eigenvalue is described by formula (37). To evaluate the normal vector $\mathbf{f}_{2}$ at this point one needs to know the associated functions $u_{1}, v_{1}$ at the double zero eigenvalue along with the eigenfunctions $u_{0}, v_{0}$. Solving at $k=2$ and $\lambda=0$ boundary value problems (12), (13) with the differential expressions and boundary forms from (38)-(41) we get

$$
\begin{gathered}
u_{1}=-\frac{\cot (b)}{6 b} x^{3}+\frac{1}{2 b^{2}} x^{2}+\frac{\cot (b)(\cos (b x)-1)+\sin (b x)}{2 b^{3}} x+ \\
+\frac{(b x-\sin (b x))(b+2 b \cos (b)-2 \sin (b))}{2 b^{4} \sin ^{2}(b)}
\end{gathered}
$$


Table 3: Splitting of the double zero eigenvalue near the singular point $\mathbf{p}_{0}=$ $(0.35431330,17.0695748)$.

\begin{tabular}{|c|c|c|}
\hline$(\Delta \eta, \Delta q)$ & $\lambda: E q \cdot(69), E q \cdot(70)$ & $\lambda: E q .(43)$ \\
\hline \multirow{2}{*}{$\left(0,10^{-4}\right)$} & $\operatorname{Re} \lambda_{1,2}=0$ & $\operatorname{Re} \lambda_{1,2}=-0.00151188$ \\
\hline & $\operatorname{Im} \lambda_{1,2}= \pm 0.32007804$ & $\operatorname{Im} \lambda_{1,2}= \pm 0.32007586$ \\
\hline \multirow{2}{*}{$\left(0,-10^{-4}\right)$} & $\lambda_{1}=0.32007804$ & $\lambda_{1}=0.32159210$ \\
\hline & $\lambda_{2}=-0.32007804$ & $\lambda_{2}=-0.31856833$ \\
\hline \multirow{2}{*}{$\left(10^{-4}, 0\right)$} & $\operatorname{Re} \lambda_{1,2}=0$ & $\operatorname{Re} \lambda_{1,2}=0.02668744$ \\
\hline & $\operatorname{Im} \lambda_{1,2}= \pm 1.55848689$ & $\operatorname{Im} \lambda_{1,2}= \pm 1.55823291$ \\
\hline \multirow{2}{*}{$\left(-10^{-4}, 0\right)$} & $\lambda_{1}=1.55848689$ & $\lambda_{1}=1.53205170$ \\
\hline & $\lambda_{2}=-1.55848689$ & $\lambda_{2}=-1.58543004$ \\
\hline \multirow{2}{*}{$-10^{-5} \mathbf{e}_{*}$} & $\lambda_{1}=-0.01207531$ & $\lambda_{1}=-0.01207543$ \\
\hline & $\lambda_{2}=-0.000431084$ & $\lambda_{2}=-0.000431085$ \\
\hline \multirow{2}{*}{$10^{-5} \mathbf{e}_{*}$} & $\lambda_{1}=0.01207531$ & $\lambda_{1}=0.01207520$ \\
\hline & $\lambda_{2}=0.000431084$ & $\lambda_{2}=0.000431082$ \\
\hline$v_{1}=\frac{x+x^{2}}{2 b^{2}}$ & \multicolumn{2}{|c|}{$-\frac{x-1}{2 b^{3}} \sin (b x)+\frac{b^{2} \cos (b)-\sin ^{2}(b)}{b^{4}(b \cos (b)-\sin (b))}$} \\
\hline
\end{tabular}

where $b=\sqrt{q_{0}}$.

Substituting eigenfunctions (62), (63) and associated function (67) into expression (45), we find the normal vector to the flutter boundary at the point $\mathbf{p}_{0}=(0.35431330,17.0695748)$

$$
\mathbf{f}_{2}=(-24288.8139,-1024.49949)
$$

Knowledge of the normal vector allows us to study the neighborhood of the point on the flutter boundary in any direction e such that $\left\langle\mathbf{f}_{2}, \mathbf{e}\right\rangle \neq 0$. In particular, for two orthogonal directions $\mathbf{e}$ $=(1,0)$ and $\mathbf{e}=(0,1)$, we get

$$
\lambda= \pm 155.848689 \sqrt{\eta_{0}-\eta}, \quad \lambda= \pm 32.0078037 \sqrt{q_{0}-q},
$$

appropriately. It is easy to see that in typical situation the double zero eigenvalue splits either into complex-conjugate pair or into two real eigenvalues, one of which is negative, Tab. 3. Thus, the 
normal vector $\mathbf{f}_{2}$ at the point $\mathbf{p}_{0}$ is directed into the divergence domain. The inequality $\left\langle\mathbf{f}_{2}, \mathbf{e}\right\rangle>0$ defines the tangent cone to this domain, and $\left\langle\mathbf{f}_{2}, \mathbf{e}\right\rangle<0$ defines the tangent cone to the flutter domain, Fig. 1. Only curves, emitted in the tangent direction to the boundary can lead to the stability domain from the singular point.

Using the degeneration condition $\left\langle\mathbf{f}_{2}, \mathbf{e}_{*}\right\rangle=0$ we choose the tangent vector $\mathbf{e}_{*}=(1,-23.7079804)$. To examine whether this vector points to the stability domain we should consider bifurcation of a double zero eigenvalue in the degenerate case. Substituting eigenfunctions (62), (63), associated functions (66), (67) and function $\hat{w}_{2}$

$$
\hat{w}_{2}=e_{2}^{*} x \frac{\cot (b)(\cos (b x)-1)+\sin (b x)}{2 b}+e_{2}^{*} \frac{b x-\sin (b x)}{2 b \sin ^{2}(b)}, \quad b=\sqrt{q_{0}}
$$

into expressions (54) we find the coefficients of equation (53)

$$
a_{1}=1250.63981, \quad a_{2}=52054.6889 .
$$

In accordance with equation (53) in the first approximation we have

$$
\lambda_{1}=1207.53146 \epsilon, \quad \lambda_{2}=43.1083501 \epsilon .
$$

It follows from (70) that the double zero eigenvalue splits into two positive simple eigenvalues ( stability) only if the parameters change in the direction specified by the vector $\mathbf{e}_{*}=(1,-23.7079804)$, Tab. 3. Changing the parameters in the opposite direction results in the splitting of the double $\lambda_{0}=0$ into two negative simple eigenvalues, which means static instability (divergence). Note that the approximate expressions for the eigenvalues are in a good agreement with the solutions of characteristic equation (43), Tab. 3.

One can see that the tangent cone to the stability domain at the singular point is a ray on the plane of parameters. Stability domain in the vicinity of this point is a long narrow tongue, Fig. 1. Our technique allows to find the quadratic approximation of the flutter and divergence domains and therefore the stability domain near the singular point.

It is easy to see, that equation (57) describing splitting of the double eigenvalue $\lambda_{0}=0$ along smooth curves tangent to the flutter boundary at the point $\mathbf{p}=\mathbf{p}_{0}$ can be rewritten as follows

$$
\lambda^{2}+\langle\mathbf{h}, \Delta \mathbf{p}\rangle \lambda+\langle\mathbf{H} \Delta \mathbf{p}, \Delta \mathbf{p}\rangle=\left\langle\mathbf{f}_{2}, \Delta \mathbf{p}\right\rangle+o\left(\|\Delta \mathbf{p}\|^{2}\right) .
$$

Components of the real vector $\mathbf{h}$ and real symmetrical matrix $\mathbf{H}$ are determined by formulae (31) and (32). Their evaluation at the singular point gives

$$
h^{1}=-917.197355, \quad h^{2}=14.0645660,
$$




$$
H^{11}=0, \quad H^{12}=-690.854898, \quad H^{22}=34.3323737 .
$$

Equation (71) provided that $\lambda=0$ gives the quadratic approximation of the divergence boundary near the singular point

$$
f_{2}^{1}\left(\eta-\eta_{0}\right)+f_{2}^{2}\left(q-q_{0}\right)=2 H^{12}\left(\eta-\eta_{0}\right)\left(q-q_{0}\right)+H^{22}\left(q-q_{0}\right)^{2}
$$

The equality of the discriminant of equation (71) to zero guarantees the nonsplitting of the double zero eigenvalue and therefore defines the approximation of the flutter boundary

$$
\begin{gathered}
f_{2}^{1}\left(\eta-\eta_{0}\right)+f_{2}^{2}\left(q-q_{0}\right)= \\
=\left(h^{1}\left(\eta-\eta_{0}\right)+h^{2}\left(q-q_{0}\right)\right)^{2} / 4-\left(2 H^{12}\left(\eta-\eta_{0}\right)\left(q-q_{0}\right)+H^{22}\left(q-q_{0}\right)^{2}\right) .
\end{gathered}
$$

Substitution of the components of the normal vector $\mathbf{f}_{2}$ from (68), the vector $\mathbf{h}$ and matrix $\mathbf{H}$ from (72) into equations (73), (74) gives the quadratic approximations of the flutter and divergence domains in the vicinity of the point $\mathbf{p}_{0}=(0.35431330,17.0695748)$. These approximations are shown on Fig. 1. by the firm thin lines. One can see that the approximation of the divergence domain is very good at the far distances from the singular point. The approximation of the flutter domain is good in the neighborhood of the point $\mathbf{p}_{0}=(0.35431330,17.0695748)$.

\section{Sensitivity analysis and stability boundaries in the problem of Smith and Herrmann}

In the previous Section we have considered the problem of stability of a cantilevered column loaded at its free end by the partially tangential follower force. T.E. Smith and G. Herrmann [23] were the first who studied the stability of such column attached to the elastic translational Winkler foundation [24] with the uniform modulus $\chi$. The differential equation of in-plane transverse vibrations of this column differs from its Beck's analogue only in the term $\kappa y(x, \tau)$, where $\kappa=\chi L_{c}^{4} / E I$. The boundary conditions remain the same.

Thus, the eigenvalue problem for the Beck column on elastic foundation looks like

$$
\begin{gathered}
u^{\prime \prime \prime \prime}+q u^{\prime \prime}+\kappa u=\lambda u, \\
U^{1}(u) \equiv u(0)=0, \\
U^{3}(u) \equiv u^{\prime \prime}(1)=0, \\
U^{2}(u) \equiv u^{\prime}(0)=0, \quad U^{4}(u) \equiv u^{\prime \prime \prime}(1)+(1-\eta) q u^{\prime}(1)=0 .
\end{gathered}
$$

The corresponding adjoint eigenvalue problem has the form

$$
l^{*}(v) \equiv v^{\prime \prime \prime \prime}+q v^{\prime \prime}+\kappa v=\lambda v
$$




$$
\begin{aligned}
& V^{1}(v) \equiv-v(0)=0, \quad V^{3}(v) \equiv v^{\prime \prime}(1)+\eta q v(1)=0 \\
& V^{2}(v) \equiv v^{\prime}(0)=0, \quad V^{4}(v) \equiv-v^{\prime \prime \prime}(1)-q v^{\prime}(1)=0
\end{aligned}
$$

The forms $V^{5}, \ldots, V^{8}$ are determined by equations (42).

Let us consider a point $\mathbf{p}_{0}=\left(\kappa_{0}, \eta_{0}, q_{0}\right)$ in the space of parameters $(\kappa, \eta, q)$, where the spectrum of eigenvalue problem (75), (76) has a real eigenvalue of multiplicity $k$ with Keldysh chain of length $k$. With the use of formulas $(22)$, (42) and $(\mathbf{7 5})-(78)$ we obtain the real vector $\mathbf{f}_{k}$

$$
\mathbf{f}_{k}=\left(\frac{\int_{0}^{1} u_{0} v_{0} d x}{\int_{0}^{1} u_{0} v_{k-1} d x}, \frac{q_{0} u_{0}^{\prime}(1) v_{0}(1)}{\int_{0}^{1} u_{0} v_{k-1} d x}, \quad \frac{\int_{0}^{1} u_{0}^{\prime \prime} v_{0} d x-\left(1-\eta_{0}\right) u_{0}^{\prime}(1) v_{0}(1)}{\int_{0}^{1} u_{0} v_{k-1} d x}\right) .
$$

We know from Section 3 of the present paper that

$$
\int_{0}^{1} u_{0} v_{0} d x=0
$$

for the eigenfunctions at the multiple eigenvalue with the Keldysh chain of length $k \geq 2$. This integral remains nonzero only if $u_{0}, v_{0}$ are the eigenfunctions of the simple eigenvalue.

Hence, the first component $f_{k}^{1}$ of the vector (79) is identically zero at any point $\mathbf{p}_{0}$, corresponding to the multiple eigenvalue $\lambda_{0}$ with the Keldysh chain. It follows from formula (21) that in such case $\lambda_{0}$ does not split in the first approximation due to variation of the modulus of elastic foundation $\kappa$

$$
\lambda=\lambda_{0}+\sqrt[k]{f_{k}^{2} \Delta \eta+f_{k}^{3} \Delta q}+o\left(\|\Delta \mathbf{p}\|^{1 / k}\right)
$$

The points $\mathbf{p}_{0}$ of the smooth parts of the flutter boundary of dimension 2 correspond to the operators $L$ with double real eigenvalues with the Keldysh chain of length 2 . From (80) it follows that the tangent plane to the flutter boundary at such points is described by the equation

$$
f_{k}^{2} \Delta \eta+f_{k}^{3} \Delta q=0
$$

and thus is always parallel to the axis $\kappa$. This is the necessary condition of independence of the flutter boundary of the elastic foundation modulus. We showed that this condition is automatically satisfied in the problem of Smith and Herrmann [23].

Recall that the authors of paper [23] came to the striking conclusion that the flutter boundary doesn't depend on the modulus of the uniform elastic foundation, analyzing the characteristic equation

$$
\Delta(\eta, q, \lambda-\kappa)=0
$$




$$
\Delta \equiv\left(2(\lambda-\kappa)+(1-\eta) q^{2}\right)(1+\cosh (a) \cos (b))+q(2 \eta-1)(q+a b \sinh (a) \sin (b)),
$$

where

$$
a=\sqrt{-\frac{q}{2}+\sqrt{\frac{q^{2}}{4}+\lambda-\kappa}}, \quad b=\sqrt{\frac{q}{2}+\sqrt{\frac{q^{2}}{4}+\lambda-\kappa}}, \quad \frac{q^{2}}{4}+\lambda-\kappa \neq 0 .
$$

Indeed, at given values of the parameters $\eta=\eta_{0}, q=q_{0}$ and real $\lambda$ the characteristic determinant is a smooth function: $\Delta=\Delta(\lambda-\kappa)$. It is clear that the zeros of the shifted function $\Delta(\lambda-\kappa)$ differ from the zeros of the function $\Delta(\lambda)$ on the amount of $\kappa$ but their multiplicities are invariable. This means that if the point $\left(0, \eta_{0}, q_{0}\right)$ is a point of the flutter boundary then the point $\left(\kappa, \eta_{0}, q_{0}\right)$ also belongs to this boundary for any $\kappa$.

On the contrary, the simple eigenvalue $\lambda_{0}=0$ becomes simple nonzero after the shift: $\lambda=\kappa$. This means that the divergence boundary should change due to variation of the elastic foundation modulus $\kappa$. This conclusion does not contradict to the sensitivity analysis because the eigenfunctions of the simple zero eigenvalue are not orthogonal and thereby the first component of the vector $\mathbf{f}_{1}$, normal to the boundary between stability and divergence domains, is not zero.

Traveling of the divergence domain. Let us find the divergence domain in the SmithHerrmann problem. Substituting $\lambda=0$ into characteristic equation (81) and expressions (82) we obtain

$$
\eta(q, \kappa)=\frac{r_{2} q-r_{1}\left(q^{2}-2 \kappa\right)}{2 r_{2} q-r_{1} q^{2}}, \quad r_{1}=1+\cosh (a) \cos (b), \quad r_{2}=q+a b \sinh (a) \sin (b) .
$$

At $\kappa=0$ the function $\eta(q)$ coincides with (44) and has its maximum $\eta_{*}=0.5$ at the values of the load parameter

$$
q_{*}=\pi^{2},(3 \pi)^{2}, \ldots,(2 j-1)^{2} \pi^{2}, \ldots
$$

At the points

$$
q_{* *}=0,(2 \pi)^{2},(4 \pi)^{2}, \ldots,(2 j)^{2} \pi^{2}, \ldots
$$

the value of the function $\eta(q)$ tends to $-\infty[17]$.

At any given $\kappa>0$ and $q>0$ the function $\eta(q)$ defined by (83) represents a smooth curve of zero eigenvalues on the plane of the parameters $\eta$ and $q$. In that case the maximum value of $\eta_{*}$ and the corresponding value of the parameter $q_{*}$ will change due to variation of the parameter $\kappa$. It is interesting to find a trajectory of the point $\left(\eta_{*}, q_{*}\right)$, say of the first maximum, on the plane of parameters $\eta, q$ due to change of the parameter $\kappa$. Solving numerically at different values of $\kappa$ the 

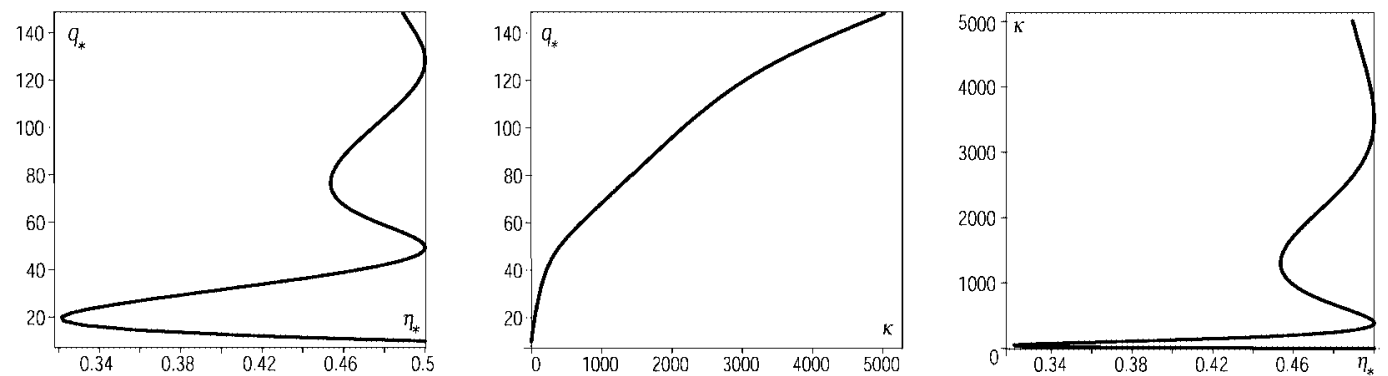

Figure 5: Changing of the point $\mathbf{p}_{*}=\left(\eta_{*}, q_{*}\right)$ due to variation of the parameter $\kappa$.

equation $d \eta / d q=0$ we can get the function $q_{*}(\kappa)$. Substituting this function into (83) we obtain the function $\eta_{*}(\kappa)$. Knowledge of these two functions allows us to find the curve $\eta_{*}\left(q_{*}\right)$, Fig. 5 .

At $\kappa=0$ we have $\mathbf{p}_{*}=(0.5,9.86960440)$. This is the point of divergence domain with the vertical tangent studied in Section 4. With the increase of $\kappa$ this point moves up and to the left until the values of $\eta_{*}=0.32112653$ and $q_{*}=19.4220703$, corresponding to $\kappa_{*}=46.4046486$. We see that the divergence domain extended to $\eta_{*}=0.5$ at $\kappa=0$ contracts with the increase of $\kappa$ to $\eta_{*}=0.32112653$. Further increase of $\kappa$ moves the point $\left(\eta_{*}, q_{*}\right)$ up and to the right expanding the divergence domain until $\eta=0.5$, then up and to the left contracting the divergence domain, and so on, Fig. 5.

Recall that the flutter domain as it was discussed earlier doesn't change with the increase of the parameter $\kappa$. Nevertheless double eigenvalues at the points of the flutter boundary change as $\lambda=\kappa$. Consider the point $\mathbf{p}_{0}=(0.35431330,17.0695748)$ on the flutter boundary where there exists a double zero eigenvalue at $\kappa=0$, see Section 5 or [17]. With the increase of $\kappa$ the double eigenvalue at $\mathbf{p}_{0}$ becomes positive, i.e. this point becomes a regular point of the stability-flutter boundary. The singularity $0^{2}$ moves out from $\mathbf{p}_{0}$. Indeed, the points on the flutter boundary situated after $\mathbf{p}_{0}$ correspond at $\kappa=0$ to the double negative eigenvalues. Since $\lambda=\kappa$ any of such negative eigenvalues becomes double zero at some $\kappa>0$. So, at $\kappa_{*}=46.4046486$ the double zero eigenvalue appears at the point $(0.32112653,19.4220703)$, studied in Section 5. At this point the flutter boundary has the vertical tangent. Therefore, with the increase of the modulus of the elastic foundation flutter boundary remains invariable while the double eigenvalues at its points change linearly with respect to $\kappa$. This has as a consequence the motion of the double zero eigenvalue along the flutter boundary. Since the curve of zero eigenvalues and the flutter boundary are tangent at the point $0^{2}$ [17], we can conclude that with a change of $\kappa$ the curve of zero eigenvalues travel up on the plane of parameters $\eta$, $q$, being always tangent to the flutter boundary. The full 


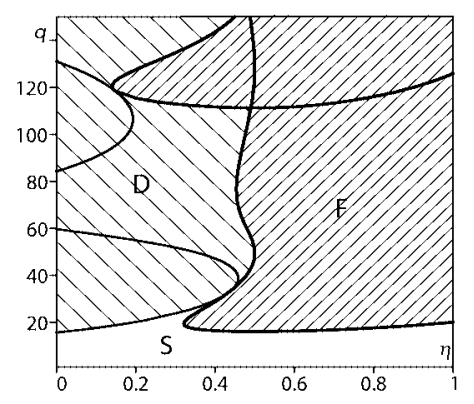

$\kappa=200$
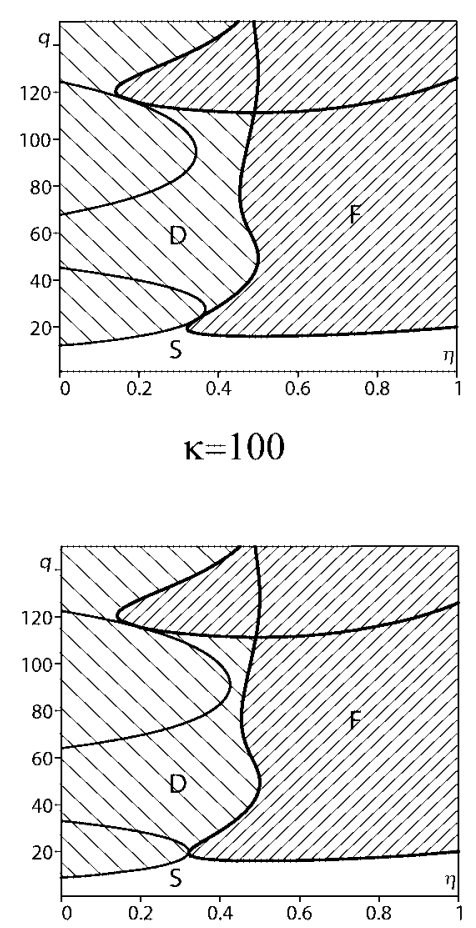

$\kappa=50$

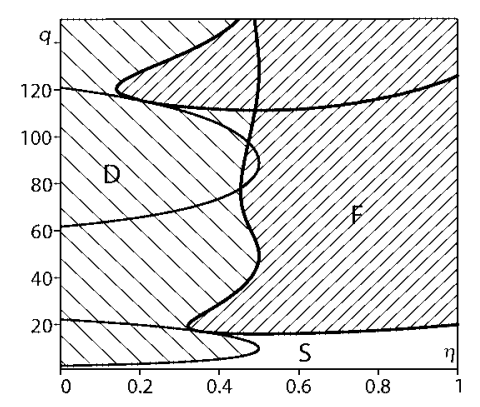

$\kappa=0$

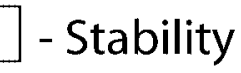

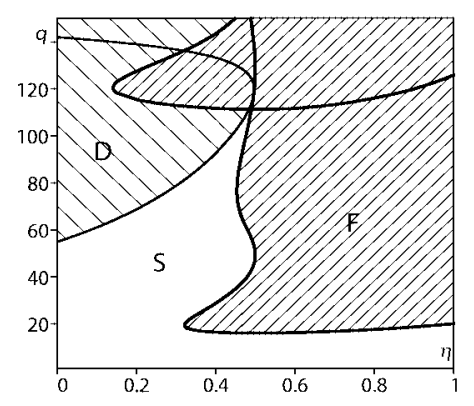

$\kappa=3000$
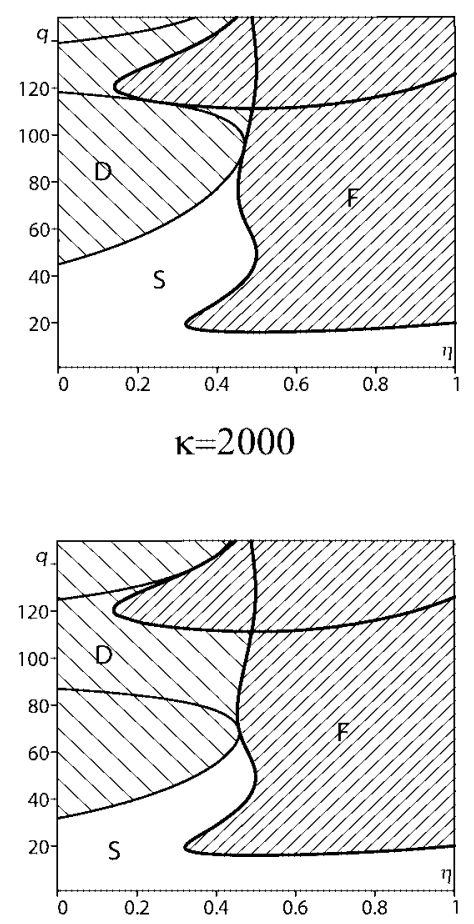

$\kappa=1000$

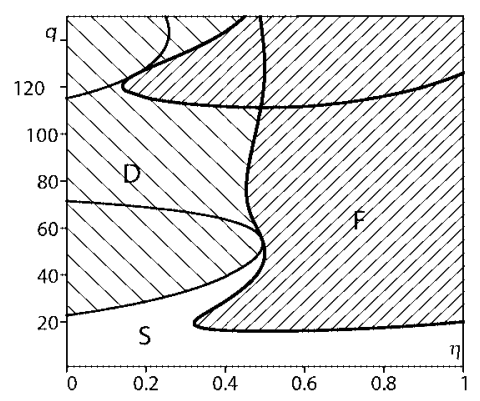

$\kappa=500$

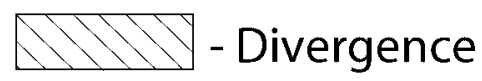

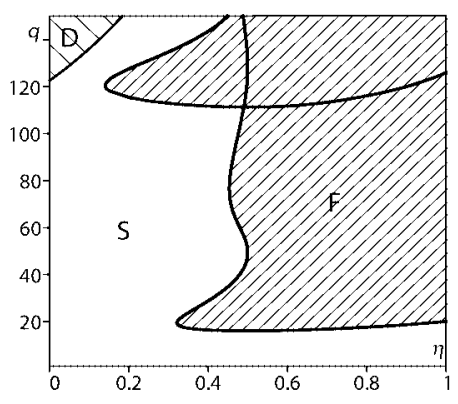

$\kappa=15000$
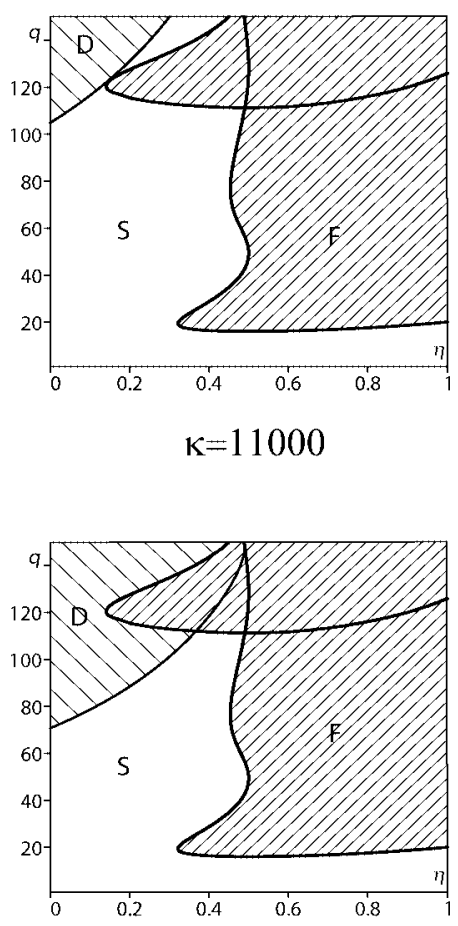

$\kappa=5000$

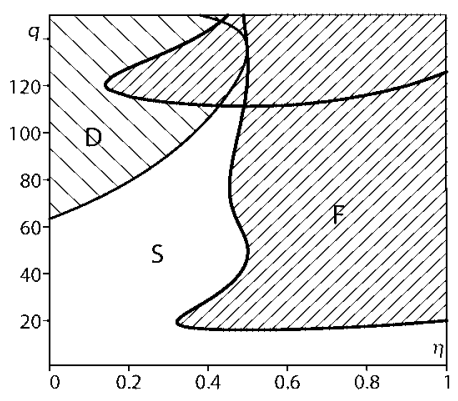

$\kappa=4000$

- Flutter

Figure 6: The travel of the divergence domain due to increase of the modulus $\kappa$. 
dynamics of the divergence boundary and the curves of zero eigenvalues is shown on Fig. 6 for $\kappa \in[0,15000]$.

On Fig. 6 stability diagrams for $\eta \in[0,1]$ and $q \in[0,150]$ are shown. One can see that in this range of parameters there are two invariable curves of double eigenvalues. The lower curve corresponds to the coincidence of the first two eigenvalues while the upper one corresponds to the collision of the third and the fourth eigenvalues. There are also two curves of zero eigenvalues for the figures corresponding to $\kappa<3000$. At the points of the lower curve the two first eigenvalues change its sign while at the points of the upper curve - the third and the fourth eigenvalue. In this sense upper and lower curves are situated at the different levels. The lower curve of zero eigenvalues is always tangent to the lower curve of double eigenvalues but can intersect the upper curve of double eigenvalues (for example at $\kappa=4000$ and $\kappa=5000$ ). And so does the upper curve of zero eigenvalues (for example at $\kappa=0$ ).

With the increase of $\kappa$ the curves of zero eigenvalues and the divergence domain together with them travel up clearing the place for the stability. At $\kappa>3000$ only the lowest curve of zero eigenvalues remains in the range $\eta \in[0,1]$ and $q \in[0,150]$. At $\kappa=15000$ the divergence is almost swept out of this range and we have on its former place a great stability domain, Fig. 6.

The idea to consider the change of stability diagram on the plane of parameters $\eta, q$ due to variation of modulus $\kappa$ seems to appear first in the work [26]. The authors of [26] noted the changing of the divergence boundary but overpassed the phenomenon of the traveling of the divergence domain along the flutter boundary and the possibility to considerably enlarge the stability domain. In the work [17] the stability diagram at $\kappa=0$ was found, but the part of the flutter boundary corresponding to the negative double eigenvalues was not shown "since it does not correspond to any physical interpretation" [17]. As one can see from Fig. 6, the introduction of another parameters $(\kappa)$ considerably changes the problem and gives a physical meaning to the "bad" part of the flutter boundary.

Sensitivity of the flutter load to the imperfections of the elastic foundation. Consider a point $\mathbf{p}_{0}=\left(\kappa_{0}, \eta_{0}, q_{0}\right)$ on the flutter boundary corresponding to the double real eigenvalue $\lambda_{0}$ with the Keldysh chain of length 2 . The critical flutter load $q_{0}$ in the problem of Smith-Herrmann does not depend on the modulus $\kappa$ of the uniform elastic foundation. Let us study the sensitivity of this load to small imperfections of the foundation. 
Namely, we consider the variation $\delta \kappa(x)$ of the modulus $\kappa$

$$
\kappa(x)=\kappa_{0}+\epsilon e^{\kappa}(x)+o(\epsilon)
$$

where $\epsilon \geq 0$ is a small parameter and $\epsilon e^{\kappa}(x)=\delta \kappa(x)$. Varying the parameters $\eta$ and $q$ in the usual form

$$
\eta=\eta_{0}+\epsilon e^{\eta}+o(\epsilon), \quad q=q_{0}+\epsilon e^{q}+o(\epsilon),
$$

substituting them as well as variation (84) into eigenvalue problem (75), (76) and taking into account expansions (9) and (16) we get two boundary value problems for determining the first coefficients of expansions (16)

$$
\begin{gathered}
w_{1}^{\prime \prime \prime \prime}+q_{0} w_{1}^{\prime \prime}+\kappa_{0} w_{1}=\lambda_{0} w_{1}+\lambda_{1} u_{0}, \\
w_{1}(0)=w_{1}^{\prime}(0)=w_{1}^{\prime \prime}(1)=w_{1}^{\prime \prime \prime}(1)+\left(1-\eta_{0}\right) q_{0} w_{1}^{\prime \prime \prime}(1)=0 ; \\
w_{2}^{\prime \prime \prime \prime}+q_{0} w_{2}^{\prime \prime}+\kappa_{0} w_{2}=\lambda_{0} w_{2}+\lambda_{1} w_{1}+\lambda_{2} u_{0}-e^{q} u_{0}^{\prime \prime}-e^{\kappa}(x) u_{0}, \\
w_{2}(0)=w_{2}^{\prime}(0)=w_{2}^{\prime \prime}(1)=w_{2}^{\prime \prime \prime}(1)+\left(1-\eta_{0}\right) q_{0} w_{2}^{\prime \prime \prime}(1)+\left[e^{q}\left(1-\eta_{0}\right)-e^{\eta} q_{0}\right] u_{0}^{\prime}(1)=0 .
\end{gathered}
$$

Taking the scalar product of equation (86) with the eigenfunction $v_{0}$ and substituting in the obtained product the solution $w_{1}$ of equation (85) we get the approximate formula describing splitting of the double eigenvalue $\lambda_{0}$

$$
\lambda=\lambda_{0} \pm \sqrt{\frac{\left(\delta \kappa(x) u_{0}, v_{0}\right)}{\left(u_{0}, v_{1}\right)}+\frac{q_{0} u_{0}^{\prime}(1) v_{0}(1)}{\left(u_{0}, v_{1}\right)} \Delta \eta+\frac{\left[\left(u_{0}^{\prime \prime}, v_{0}\right)-\left(1-\eta_{0}\right) u_{0}^{\prime}(1) v_{0}(1)\right]}{\left(u_{0}, v_{1}\right)} \Delta q},
$$

where $\Delta \eta=\eta-\eta_{0}$ and $\Delta q=q-q_{0}$. The double eigenvalue does not split in the first approximation due to change of parameters if the radicand in (87) is zero. Thus, if we wish to change the parameters $\kappa$ and $\eta$ to obtain the critical flutter load different from $q_{0}$ we should impose the nonsplitting condition on their variations

$$
\Delta q=-\frac{\left(\delta \kappa(x) u_{0}, v_{0}\right)}{\left(u_{0}^{\prime \prime}, v_{0}\right)-\left(1-\eta_{0}\right) u_{0}^{\prime}(1) v_{0}(1)}-\frac{q_{0} u_{0}^{\prime}(1) v_{0}(1)}{\left(u_{0}^{\prime \prime}, v_{0}\right)-\left(1-\eta_{0}\right) u_{0}^{\prime}(1) v_{0}(1)} \Delta \eta .
$$

Formula (88) clearly shows that the critical flutter load is sensitive to changes in the parameter $\eta$ as well as in parameter $\kappa$ if this parameter is distributed. If $\delta \kappa=$ const., then $\int_{0}^{1} \delta \kappa u_{0} v_{0} d x=$ $\delta \kappa \int_{0}^{1} u_{0} v_{0} d x=0$ and the flutter load does not depend on the modulus of the elastic foundation. 


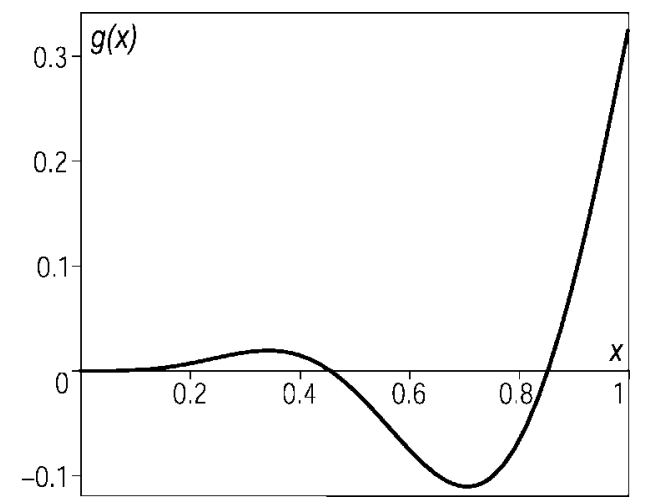

Figure 7: Gradient function $g(x)$ of the critical flutter load w.r.t. the elastic foundation modulus distribution $\kappa(x)$.

Suppose that the parameter $\eta=\eta_{0}$ is fixed. Introducing the gradient function

$$
g(x)=\frac{-u_{0}(x) v_{0}(x)}{\int_{0}^{1} u_{0}^{\prime \prime} v_{0} d x-\left(1-\eta_{0}\right) u_{0}^{\prime}(1) v_{0}(1)}
$$

we can write

$$
\Delta q=\int_{0}^{1} g(x) \delta \kappa(x) d x .
$$

Consider, for example, the Beck point ( $\left.\kappa_{0}=0, \eta_{0}=1, q_{0}=20.0509536\right)$. Substituting into (89) the eigenfunctions (46), (47) evaluated at that point we get the gradient of the critical flutter load $q_{0}$ with respect to the distributed elastic foundation modulus $\kappa(x)$, Fig. 7 .

One can see from Fig. 7 that the tip of the Beck column is very sensitive to the presence of the elastic foundation. That fact is in accordance with the result of the work [18] where the high influence of the end spring stiffness on the flutter load of the Beck column was shown.

Let us take a variation of $\kappa$ in the gradient direction: $\delta \kappa(x)=\gamma g(x)$. Substituting $\delta \kappa(x)$ into (90) we get

$$
q=q_{0}+\gamma \int_{0}^{1} g^{2}(x) d x=q_{0}+\gamma 0.00708579 .
$$

The obtained formula shows that the breakdown of the uniformity of the elastic foundation can increase $(\gamma>0)$ or decrease $(\gamma<0)$ the critical flutter load. That fact, firstly observed in the work [25], stimulated the investigation of different types of distributed elastic foundations, for example partial elastic foundation [27]. It seems that the choice of the model of elastic foundation, among which the translational Winkler foundation is the simplest one [24], is the key to the paradox of Smith-Herrmann. 
Note on a parasite solutions of characteristic equation (81). Consider the relationship

$$
\lambda=\kappa-\frac{q^{2}}{4} .
$$

Such defined $\lambda$ are the roots of characteristic equation (81). Indeed, taking into account (91) we can transform (81) into

$$
\frac{q^{2}}{2}(1-2 \eta)\left(\cos ^{2}\left(\sqrt{\frac{q}{2}}\right)+\sin ^{2}\left(\sqrt{\frac{q}{2}}\right)-1\right) \equiv 0 .
$$

Consequently, equation $\kappa=q^{2} / 4$ gives a curve of zero eigenvalues on the parameter plane $(\kappa, q)$ and can be the boundary between stability and divergence domains.

Let us show that for $q \geq 0$ the eigenfunctions at the eigenvalues $\lambda$ defined by (91) are $u(x) \equiv 0$ and therefore these solutions have no influence on the stability of the column. Substitution of (91) into expressions (82) gives

$$
b=-i a=\sqrt{\frac{q}{2}} .
$$

In this case the general solution of equation $(75)$ has the form

$$
u(x)=C_{1} \cos \left(x \sqrt{\frac{q}{2}}\right)+C_{2} \sin \left(\sqrt{\frac{q}{2}}\right)+C_{3} x \cos \left(x \sqrt{\frac{q}{2}}\right)+C_{4} x \sin \left(x \sqrt{\frac{q}{2}}\right) .
$$

Substituting this general solution into boundary conditions (76) we get the system of linear equations on the coefficients $C_{i}, i=1 \ldots 4$. This system has a nontrivial solution if and only if its determinant

$$
\Delta(\eta, q)=\frac{q^{2}}{8}\left((2 \eta-1)\left(q+2 \sin ^{2}\left(\sqrt{\frac{q}{2}}\right)\right)+4+4 \cos ^{2}\left(\sqrt{\frac{q}{2}}\right)\right)
$$

is equal to zero.

From (92) one can see that for $\eta \geq 0.5$ the equation $\Delta(q)=0$ has no solutions $q>0$, i.e. all the coefficients $C_{i}=0$ and $u(x) \equiv 0$. The only root is $q=0$. But in this case the general solution has another form

$$
u(x)=C_{1}+C_{2} x+C_{3} x^{2}+C_{4} x^{3} .
$$

Substituting it into the boundary conditions (76) it is easy to determine that $u(x) \equiv 0$. The case $q>0, \eta<0.5$ needs further investigation. 


\section{Stability of a panel at high supersonic speeds}

As another example we consider a simply-supported panel of the uniform thickness $h$, length $a_{1}$ and width $a_{2}$ with air flowing above at supersonic Mach number $M$. The panel has a density $\mu$ and stiffness $D=E h^{3} / 12\left(1-\nu^{2}\right)$, where $E$ is the Young modulus and $\nu$ is the Poisson number. The speed of sound in an undisturbed gas medium of density $\rho_{0}$ is designated by $c_{0}$. The panel is subjected to constant midplane force intensities $N_{1}$ and $N_{2}$ (positive in compression). This problem was considered first in 1950-s [28, 29, 30] and still remains important [31], [32].

Introducing the non-dimensional variables: $k=a_{1} / a_{2}$, deflection $z$, coordinates $x, y$, time $\tau=t / \sqrt{\mu h a_{1}^{4} / D}$, dynamical pressure $p$ and forces $q, r$

$$
z=\frac{Z}{a_{1}}, \quad x=\frac{X}{a_{1}}, \quad y=\frac{Y}{a_{2}}, \quad p=\frac{\rho_{0} c_{0} M^{2} a_{1}^{3}}{D \sqrt{M^{2}-1}}, \quad q=\frac{N_{1} a_{1}^{2}}{D}, \quad r=\frac{N_{2} a_{1}^{2}}{D},
$$

we get the differential equation of small vibrations of the panel [29, 30]

$$
\frac{\partial^{4} z}{\partial x^{4}}+2 k^{2} \frac{\partial^{4} z}{\partial x^{2} \partial y^{2}}+k^{4} \frac{\partial^{4} z}{\partial y^{4}}+q \frac{\partial^{2} z}{\partial x^{2}}+r k^{2} \frac{\partial^{2} z}{\partial y^{2}}+p \frac{\partial z}{\partial x}+\frac{\partial^{2} z}{\partial \tau^{2}}=0
$$

with the boundary conditions

$$
\begin{aligned}
& z(0, y, \tau)=z_{x x}(0, y, \tau)=z(1, y, \tau)=z_{x x}(1, y, \tau)=0 \\
& z(x, 0, \tau)=z_{y y}(x, 0, \tau)=z(x, 1, \tau)=z_{y y}(x, 1, \tau)=0 .
\end{aligned}
$$

We seek the solution of $(93)$ in the form

$$
z(x, y, \tau)=u(x) \sin (n \pi y) \exp (i \sqrt{\lambda} \tau)
$$

Substituting (95) into equation (93) and boundary conditions (94) we come to the eigenvalue problem, see [29]

$$
\begin{gathered}
u^{\prime \prime \prime \prime}+\left(q-2 n^{2} \pi^{2} k^{2}\right) u^{\prime \prime}+p u^{\prime}+\left(n^{4} \pi^{4} k^{4}-n^{2} \pi^{2} k^{2} r\right) u=\lambda u \\
u(0)=0, \quad u^{\prime \prime}(0)=0, \quad u(1)=0, \quad u^{\prime \prime}(1)=0 .
\end{gathered}
$$

Consider first the panel of infinite span $(k \rightarrow 0)$. In this particular case equations $(\underline{96})$ take a simpler form and the eigenvalue problem looks like

$$
\begin{gathered}
l(u)=u^{\prime \prime \prime \prime}+q u^{\prime \prime}+p u^{\prime}=\lambda u, \\
U^{1}(u) \equiv u(0)=0, \quad U^{2}(u) \equiv u^{\prime \prime}(0)=0 \\
U^{3}(u) \equiv u(1)=0, \quad U^{4}(u) \equiv u^{\prime \prime}(1)=0 .
\end{gathered}
$$


For the problem adjoint to (97), (98) we have

$$
\begin{gathered}
l^{*}(v)=v^{\prime \prime \prime \prime}+q v^{\prime \prime}-p v^{\prime}=\lambda v, \\
V^{1}(v) \equiv v(0)=0, \quad V^{2}(v) \equiv v^{\prime \prime}(0)=0, \\
V^{3}(v) \equiv v(1)=0, \quad V^{4}(v) \equiv v^{\prime \prime}(1)=0 .
\end{gathered}
$$

Eigenfunction $v(x)$ of adjoint eigenvalue problem (99), (100) can be expressed through the eigenfunction $u(x)$ of eigenvalue problem (97), (98)

$$
v(x)=\gamma u(1-x)
$$

where $\gamma$ is a nonzero constant.

A general solution of equation (97) is a linear combination of exponents $e^{s x}$. Substituting $e^{s x}$ into (97) we get the polynomial equation on $s$

$$
P(s) \equiv s^{4}+q s^{2}+p s-\lambda=0 .
$$

The roots of the polynomial $P(s)$ satisfy the following conditions:

$$
\begin{gathered}
s_{1}+s_{2}+s_{3}+s_{4}=0, \quad s_{1} s_{2}+s_{3} s_{4}+\left(s_{1}+s_{2}\right)\left(s_{3}+s_{4}\right)=q, \\
s_{1} s_{2}\left(s_{3}+s_{4}\right)+\left(s_{1}+s_{2}\right) s_{3} s_{4}=-p, \quad s_{1} s_{2} s_{3} s_{4}=-\lambda .
\end{gathered}
$$

We will further consider only nonnegative values of the parameter $q$. To find the boundaries of flutter and divergence domains it is sufficient to know only real eigenvalues $\lambda$.

If $q>0$ and $\lambda>0$, then two roots $s_{1}, s_{2}$ of (101) are real and two others form a complexconjugate pair: $s_{3,4}=a \pm i b$. It follows from (102) that the roots $s_{1}, s_{2}$ and the parameters $p, q$ can be expressed through $a, b$ and $\lambda$

$$
\begin{aligned}
& s_{1,2}=-a \pm A, \quad A=\sqrt{a^{2}+\frac{\lambda}{a^{2}+b^{2}}}, \\
& p=2 a\left(b^{2}+A^{2}\right), \quad q=b^{2}-2 a^{2}-A^{2} .
\end{aligned}
$$

It is clear that the general solution taken in the form

$$
u(x)=C_{1} e^{-a x} \sinh (A x)-C_{2} e^{-a x} \cosh (A x)+C_{2} e^{a x} \cos (b x)+C_{3} e^{a x} \sin (b x)
$$


satisfies the first of boundary conditions (98). Substituting it in the remaining boundary conditions we obtain the system of three linear equations with respect to the variables $C_{1}, C_{2}, C_{3}$. This system has a nontrivial solution iff the determinant $\Delta$ of the system matrix is equal to zero:

$$
\begin{gathered}
\Delta(a, p, q)=0, \\
\Delta \equiv\left(\left(A^{2}+b^{2}\right)^{2}+4 a^{2}\left(b^{2}-A^{2}\right)\right) \sinh (A) \sin (b)-8 A a^{2} b(\cosh (A) \cos (b)-\cosh (2 a)),
\end{gathered}
$$

where

$$
b=\sqrt{\frac{p}{4 a}+\frac{q}{2}+a^{2}}, \quad A=\sqrt{\frac{p}{4 a}-\frac{q}{2}-a^{2}} .
$$

Equations (105) and (106) give $a$ as a function of the parameters $p$ and $q$. Substituting $a(p, q)$ into the equation

$$
\lambda=\frac{p^{2}}{16 a^{2}}-\left(2 a^{2}+\frac{q}{2}\right)^{2}
$$

we get the eigenvalue $\lambda(p, q)$, see [29]. Relationships (106) along with equation (107) are the consequence of equations (103).

To find the flutter boundary numerically we should change one of the parameters $p$ or $q$ while remaining parameter is fixed until two real eigenvalues collide with origination of a double real eigenvalue. The equation describing the divergence boundary [30] follows from (105]), if we put $A=a$ in it

$$
\begin{gathered}
\left(\left(a^{2}+b^{2}\right)^{2}+4 a^{2}\left(b^{2}-a^{2}\right)\right) \sinh (a) \sin (b)-8 a^{3} b(\cosh (a) \cos (b)-\cosh (2 a))=0, \\
a=q G^{-1 / 3}-\frac{1}{12} G^{1 / 3}, \quad b=\sqrt{3}\left(a+\frac{1}{6} G^{1 / 3}\right), \quad G=-108 p+12 \sqrt{12 q^{3}+81 p^{2}} .
\end{gathered}
$$

The results of numerical analysis are presented on Fig. 8. One can see that the plane of parameters $p$ and $q$ is subdivided into stability $(\mathrm{S})$, flutter $(\mathrm{F})$ and divergence $(\mathrm{D})$ domains, which are symmetric with respect to the vertical axis. This is the consequence of the symmetric boundary conditions (98).

The boundary of the stability domain is nonsmooth and has two singular points $0^{2}$. To obtain the exact coordinates of these points we need to move along the curve (108), the part of which describes the boundary between stability and divergence domains, until the integral $\int_{0}^{1} u_{0}(x) v_{0}(x) d x$ calculated at the points of this boundary becomes zero. The eigenfunctions of eigenvalue problems (97)-(100), corresponding to the double eigenvalue $\lambda=0$, have the form

$$
u_{0}(x)=K_{1} e^{a x} \sin b x-K_{2} e^{-a x} \sinh (a x)+e^{a x} \cos (b x)-e^{-a x} \cosh (a x),
$$




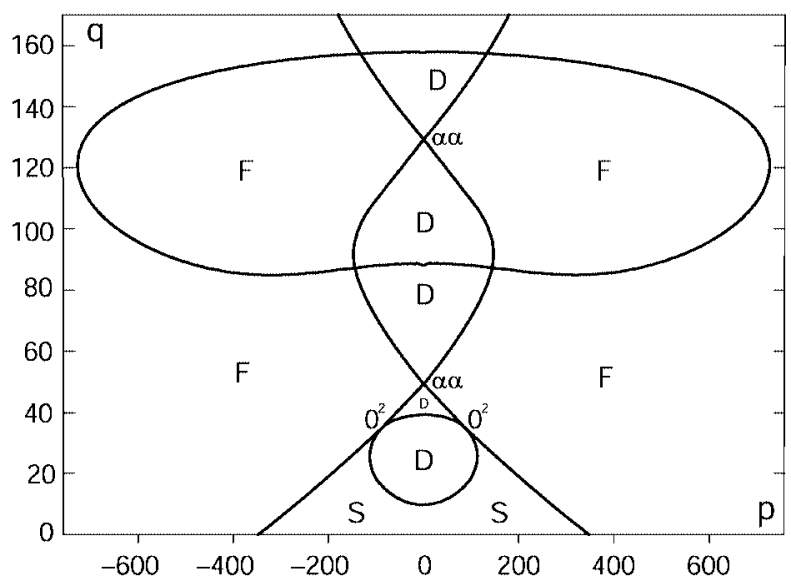

Figure 8: Stability Diagram.

$$
v_{0}(x)=u_{0}(1-x)
$$

where the coefficients $K_{1}$ and $K_{2}$ are as follows

$$
\begin{gathered}
K_{1}=\frac{\left(a^{2}+b^{2}\right) \sinh (a) e^{-a}+2 a^{2}\left(\cosh (a) e^{-a}-\cos (b) e^{a}\right)}{2 a\left(b \sinh (a) e^{-a}+a \sin (b) e^{a}\right)}, \\
K_{2}=\frac{\left(a^{2}+b^{2}\right) \sin (b) e^{a}-2 a b\left(\cosh (a) e^{-a}-\cos (b) e^{a}\right)}{2 a\left(b \sinh (a) e^{-a}+a \sin (b) e^{a}\right)} .
\end{gathered}
$$

Evaluating the scalar product of eigenfunctions (109), (110) we obtain the coordinates of the singular points

$$
\left(p_{0}= \pm 90.1406979, q_{0}=34.3998023\right) \text {. }
$$

These coordinates are in agreement with the coordinates $\left(p_{0}= \pm 88, q_{0}=34\right)$ found in the book [30] with the use of the Galerkin method.

The stability diagram also shows the nonsmoothness of the flutter boundary. It has singularities $\alpha \alpha$ [22] at $p=0$. These singularities correspond to the semisimple double real eigenvalues $\alpha$ with the two eigenfunctions. Note that in the absence of the flow $(p=0)$ eigenvalue problem (97), (98) is selfadjoint and its spectrum is free from the multiple eigenvalues with the Keldysh chains of length $k \geq 2$.

Double semisimple eigenvalues were investigated in the work [4]. It was shown that the corresponding singularity is a cone in the space of three parameters. On the plane of two parameters we have only the sections of the cone which in generic case are hyperbolae, parabolae and ellipses. Two intersecting lines in the section of the cone appear in non-generic situations. In our problem the non-genericity is caused by the symmetric boundary conditions. It is interesting to break down the symmetry considering another boundary conditions for the plate in the supersonic flow. 


\section{Conclusion}

This work has presented an approach allowing to obtain explicit formulae for the bifurcation of multiple eigenvalues of non-selfadjoint differential operators smoothly dependent on a vector of real parameters. The formulae found use the derivatives of the differential expression and the boundary forms with respect to parameters as well as the functions of the Keldysh chain evaluated at the point of the parameter space corresponding to a multiple eigenvalue. It is shown with their use that the "flutter condition" for the pure circulatory systems is a simple consequence of the existence of the Keldysh chain of length $k \geq 2$.

The results obtained let us to study the splitting of the multiple eigenvalues both in regular and degenerate cases and serve as a basis for the sensitivity analysis of continuous non-conservative systems, allowing to avoid the variational calculus in every particular problem to find sensitivities of eigenvalues or critical values of parameters.

The advantages of the proposed approach are illustrated by the mechanical examples, including the extended Beck problem, the paradox of Smith-Herrmann, and the problem of stability of a plate in supersonic flow. With the use of the bifurcation analysis of eigenvalues stability boundaries of these problems are investigated. The singularities of different types are found and linear and quadratic approximations to the stability and instability domains both at regular and singular points of their boundaries are constructed.

In the problem of Smith-Herrmann the phenomenon of traveling of the divergence domain due to change of the elastic foundation modulus is found and discussed in detail.

Acknowledgement. The authors are grateful to Wolfhard Kliem and Pauli Pedersen for fruitful discussions on different aspects of this paper. The first author also thanks INTAS for the support of his work by the Young Scientists Fellowship No. 01/1-057.

\section{References}

[1] Keldysh M.V. On Eigenvalues and Eigenfunctions of Some Classes of Nonselfadjoint Equations. Doklady AN SSSR 1951; 77(1): 11-14. (in Russian)

[2] Seyranian A.P. Sensitivity Analysis of Multiple Eigenvalues. Mechanics of Structures and Machines 1993; 21(2): 261-284. 
[3] Mailybaev A.A., Seyranian A.P. On Singularities of a Boundary of the Stability Domain. SIAM J. Matrix Anal. Appl. 2000; 21(1): 106-128.

[4] Seyranian A.P., Kliem W. Bifurcations of Eigenvalues of Gyroscopic Systems with Parameters near Stability Boundaries. Transactions of the ASME. Journal of Applied Mechanics. 2001; 68(2): 199-205.

[5] Naimark M.A. Linear Differential Operators. Part I. Frederick Ungar Publishing Co.: New York, 1967.

[6] Vishik M.I. and Lyusternik L.A. Solution of Some Perturbation Problems in the Case of Matrices and Selfadjoint or Non-selfadjoint Equations. Russian Math. Surveys 1960; 15(3): 1-73.

[7] Gohberg I.C., Krein M.G. Introduction to the Theory of Linear Nonselfadjoint Operators. American Mathematical Society: Providence, RI, 1969.

[8] Plaut, R.H. Determining the Nature of Instability in Nonconservative Problems. AIAA J. 1972; 10: 967-968.

[9] Bolotin V.V. Non-conservative Problems of the Theory of Elastic Stability. Pergamon Press: Oxford, 1963.

[10] Ziegler H. Principles of Structural Stability. Blaisdell. Waltham: Mass., 1968.

[11] Leipholz H. Stability Theory. John Wiley \& Sons and B.G. Teubner: Stuttgart, 1987.

[12] Seyranian A.P., Kirillov O.N. Bifurcation Diagrams and Stability Boundaries of Circulatory Systems. Theoretical and Applied Mechanics. 2000; 26: 135-168.

[13] Teytel M. How Rare are Multiple Eigenvalues? Communications on Pure and Applied Mathematics. 1999; 52(8): 917-934

[14] Hartman P. Ordinary Differential Equations. Second Edition. Birkhauser: Boston, Basel, Stuttgart, 1982.

[15] Chow C.-N., Hale J.K. Methods of Bifurcation Theory. Grundlehren der mathematischen Wissenschaften 251. Springer-Verlag: New York. Berlin. Heidelberg, 1982.

[16] Beck M. Die Knicklast des einseitig eingespannten, tangential gedruckten Stabes. ZAMM 1952; 3: 225-228. 
[17] Kordas Z. and Zyczkowsky M. On the Loss of Stability of a Rod under a Super-tangential Force. Archiwum Mechaniki Stosowanej. 1963; 15(1): 7-31.

[18] Pedersen P. Influence of Boundary Conditions on the Stability of a Column under Nonconservative Load. Int. J. Solids Structures. 1977; 13(5): 445-455.

[19] Pedersen P., Seyranian A.P. Sensitivity Analysis for Problems of Dynamic Stability. Int. J. Solids Structures. 1983; 19(4): 315-335.

[20] Andreichikov I.P., Yudovich V.I. On the Stability of Viscoelastic Rods. Mekhanika Tverdogo Tela. 1974; (2): 78-87. (in Russian)

[21] Kirillov O.N., Seyranian A.P. Overlapping of Frequency Curves in Nonconservative Systems. Doklady Physics. 2001; 46(3): 184-189.

[22] Arnold V.I. Geometrical Methods in the Theory of Ordinary Differential Equations. Springer Verlag: New York and Berlin, 1983.

[23] Smith T.E., Herrmann G. Stability of a Beam on an Elastic Foundation Subjected to a Follower Force. Transactions of the ASME. Journal of Applied Mechanics. 1972; 39(2): 628-629.

[24] Kerr A.D. On the Formal Development of Elastic Foundation Models. Ingenieur-Archiv. 1984; 54: 455-464.

[25] Sundararajan C. Stability of Columns on Elastic Foundations Subjected to Conservative and Non-conservative Forces. Journal of Sound and Vibration. 1974; 37(1): 79-85.

[26] Lee S.Y., Lin J.C., Hsu K.C. Elastic Instability of a Beam Resting on an Elastic Foundation Subjected to a Partially Tangential Force. Computers and Structures. 1996; 59(6): 983988.

[27] Elishakoff I., Impollonia N. Does a Partial Elastic Foundation Increase Flutter Velocity of a Pipe Conveying Fluid? Transactions of the ASME. Journal of Applied Mechanics. $2001 ; \mathbf{6 8}(2): 206-212$.

[28] Hedgepeth J.M. On the Flutter of Panels at High Mach Numbers. Journal of the Aeronautical Sciences 1956; 23(6): 609-610.

[29] Hedgepeth J.M. Flutter of Rectangular Simply Supported Panels at High Supersonic Speeds, Journal of the Aeronautical Sciences 1957; 24(8): 563-573, 586. 
[30] Vol'mir A.S. Stability of Elastic Systems. ENTIS: Virginia, 1965.

[31] Jørgensen O. Optimization of the Flutter Load by Material Orientation. Mechanics of Structures and Machines. 1991; 19(3): 411-436.

[32] Pidaparti R.M.V., Yang H.T.Y. Supersonic Flutter Analysis of Composite Plates and Shells. AIAA Journal. 1993; 31(6): 1109-1117. 